I. Grundlagen 


\title{
Historischer Überblick über die rechtliche Lage polnischer Arbeitnehmer in Deutschland
}

\author{
Herbert Szurgacz
}

Vorbemerkungen

A. Die rechtliche Lage polnischer Arbeiter in Deutschland bis 1918

I. Die Rekrutierung polnischer Landarbeiter nach Deutschland

II. Rechtliche Grundlagen der Gestaltung der Arbeits- und Lebensbedingungen polnischer Landarbeiter

III. Die rechtliche Lage der polnischen Landarbeiter während des 1. Weltkrieges 30

IV. Die Ruhrpolen in den Industriegebieten Preußens

B. Die rechtliche Lage der polnischen Arbeiter in der Weimarer Republik 32

\begin{tabular}{ll} 
I. Vorbemerkungen & 32 \\
\hline
\end{tabular}

II. Die arbeitsrechtliche Lage der polnischen Landarbeiter 33

III. Gestaltung der sozialversicherungsrechtlichen Verhältnisse 36

C. Die rechtliche Lage der polnischen Arbeiter im nationalsozialistischen

Staat 1933-1945

I. Der Nationalsozialismus und das Arbeits- und Sozialversicherungsrecht 1933-1939

II. Die Rechtslage der polnischen Arbeiter in der Zeit 1939-1945 im Reich und in den in das Reich eingegliederten Ostgebieten

1. Die politischen Ziele der Naziherrschaft gegenüber Polen

2. Über das Recht der Zwangsarbeit gegenüber Polen im Allgemeinen

3. Einige Institutionen des Rechts der Zwangsarbeit

a) Entstehung und Auflösung des Beschäftigungsverhältnisses

b) Erholungsurlaub

c) Den polnischen Arbeitern gegenüber angewandtes Maßnahmensystem

d) Die Sicht der rechtlichen Lage der polnischen Zwangsarbeiter in der rechtswissenschaftlichen Literatur

4. Die Lage der polnischen Arbeiter in der Sozialversicherung

D. Die arbeits- und sozialversicherungsrechtliche Lage der polnischen

Arbeitnehmer in Deutschland 1946-1994

I. Die Nachkriegszeit

II. Die Zeit der Normalisierung 1970-1989 57

III. Übergang zur Zusammenarbeit

Schlussbemerkungen 


\section{Vorbemerkungen}

Die Beschäftigung polnischer Arbeiter in Deutschland hat eine lange Geschichte. Als Massenerscheinung hat sie in den 80er Jahren des 19. Jahrhunderts begonnen. Sie war Teilbereich der allgemeineren Erscheinung einer Erwerbsmigration der Polen in verschiedene Länder des damaligen Europa und nach Amerika, die durch unterschiedliche Faktoren verursacht wurde. Insbesondere waren es die ungünstige Agrarstruktur, die ländliche Überbevölkerung, welche durch die Landwirtschaft nicht mehr aufzufangen war, und der Mangel an Industrialisierung im damals geteilten Polen. Auf diese Aspekte möchte ich an dieser Stelle jedoch nicht eingehen.

Einer Klärung bedarf der zeitliche, persönliche und sachliche Rahmen dieser Abhandlung. Selbst der Begriff polnischer Arbeiter ist nicht eindeutig. Er könnte nämlich als eine soziokulturelle und ethnische Kategorie verstanden werden, d.h. der Pole als Mitglied der polnischen Nation, oder auch im staatsrechtlichen Sinne - Pole als Bürger des polnischen Staates. In dem zweitgenannten Sinn sollten die Erwägungen eigentlich erst die Zeit nach 1918 betreffen, d.h. nach der Erreichung der Unabhängigkeit durch Polen, das bis dahin unter Preußen, Russland und Österreich aufgeteilt war. Jedoch spricht Einiges dafür, den zeitlichen Rahmen bis in die 80er Jahre des 19. Jahrhunderts $\mathrm{zu}$ verschieben. In dieser Zeit waren schon tausende sog. Russland- und ÖsterreichUngarn-Polen in Deutschland als Landarbeiter tätig. In den deutschen Industriegebieten, insbesondere im Ruhrgebiet, haben damals auch tausende deutsche Staatsangehörige mit polnischem Wurzeln aus den östlichen Provinzen Preußens, insbesondere aus der Provinz Posen und Oberschlesien, sog. Ruhrpolen, eine Beschäftigung aufgenommen. Einige der in dieser Zeit eingeführten Maßnahmen haben nach 1918, wenn auch in einer anderen Form, weiter gegolten. Einige damals entstandene soziale Probleme bedurften einer Lösung, die man in der Zeit nach 1918 gefunden hat.

Diese Umstände sprechen dafür, den zeitlichen Rahmen bis in die 80er Jahre des 19. Jahrhunderts zu verschieben. Die Tatsache, dass sich in dieser Zeit das Arbeitsrecht in der Industrie in der Anfangsphase befand und in der Landwirtschaft die Verhältnisse zwischen Landarbeitern und den Gutsbesitzern noch durch feudale Merkmale gekennzeichnet waren, sollte dem nicht entgegenstehen. Mein Interesse wird sich auf den Bereich des Arbeits- und Sozialrechts konzentrieren. Die rechtliche Lage der polnischen Arbeitnehmer in anderen Bereichen, wie z.B. im Steuerrecht, im Strafrecht bzw. im Zivilrecht wird hier grundsätzlich nicht behandelt. Im Rahmen dieses Aufsatzes wird es nur im begrenzten Umfang möglich sein, den allgemeineren Hintergrund, d.h. u.a. die wirtschaftlichen, politischen, soziokulturellen und verfassungsrechtlichen Faktoren, die gleichzeitig auf die rechtlichen Entwicklungen Einfluss genommen haben, darzustellen.

Was den zweiten zeitlichen Eckpunkt dieses Aufsatzes betrifft, soll es die Assoziierung Polens mit den Europäischen Gemeinschaften im Jahre 1994 sein. 


\section{A. Die rechtliche Lage polnischer Arbeiter in Deutschland bis 1918}

\section{Die Rekrutierung polnischer Landarbeiter nach Deutschland}

In den 80er Jahren des 19. Jahrhunderts verstärkten sich in Deutschland die Probleme, die mit dem Arbeitermangel verbunden waren. Ein schneller Prozess der Industrialisierung im Ruhrgebiet und die Auswanderung der ostelbischen Landarbeiterbevölkerung in die Industriegebiete sowie nach Amerika haben einen Bedarf an Arbeitskräften hervorgerufen, der durch ausländische Arbeiter aus verschiedenen Teilen Europas, darunter auch durch Polen aus den durch Russland und Österreich besetzten Gebieten befriedigt werden sollte. Viele ostdeutsche Gutsbesitzer haben mit der Rekrutierung der polnischen Arbeitskräfte begonnen. Es wanderten aus dem durch Russland okkupierten Polen nach Deutschland, überwiegend in die preußischen Gebiete

$$
\begin{aligned}
& 1890 \text { - } 17.000 \\
& 1895 \text { - } 156.000 \\
& 1899 \text { - } 109.000 \\
& 1905 \text { - } 397.000 \text { polnische Landarbeiter ab. }{ }^{1}
\end{aligned}
$$

Gemäß den amtlichen Statistiken des preußischen Staates lebten Mitte des 19. Jahrhunderts in den Provinzen Rheinland-Westfalen insgesamt 16.000 Polen. Im Jahre 1890 sollten es dagegen mehr als 30.000, im Jahre 1910 schon um 300.000 und vor dem 1. Weltkrieg zwischen 350.000 und 450.000 sog. Ruhrpolen gewesen sein. ${ }^{2}$ Im Gegensatz zu den polnischen Landarbeitern stammten sie aus dem Osten des Deutschen Reiches, d.h. den östlichen Provinzen Preußens, insbesondere aus der Provinz Posen und Oberschlesien, und waren preußische Bürger. Was die faktische Lage, die Organisation des täglichen Lebens wie auch die rechtliche Position anbelangt, unterschieden sie sich grundsätzlich von den polnischen Landarbeitern, deren Lage hier an erster Stelle behandelt wird.

Die Anwerbung polnischer Landarbeiter nach Deutschland ging mit einem Konflikt zwischen den wirtschaftlichen und politischen Interessen einher. Sie war auch in Preußen Gegenstand ständiger Diskussionen und Auseinandersetzungen, deren Gegenstand die Sorge um die Verdrängung der Deutschen durch die Polen war. ${ }^{3}$ Sie führten 1885 zunächst zu der Einführung von Vorschriften, die zum Ziel hatten, die Zuwanderung von Auslandspolen zu mindern. Diese Begrenzungen konnten jedoch nicht lange gegen

1 Von Schulz, Die rechtliche Stellung der ausländischen Landarbeiter in Deutschland, 1939, S. 2; Herbert, Geschichte der Ausländerbeschäftigung in Deutschland 1880 bis 1980. Saisonarbeiter, Zwangsarbeiter, Gastarbeiter, 1986, S. 27; Lazinka, Wychodźtwo polskie w Westfalii i Nadrenii 1890-1923 (Die polnische Auswanderung in Westfalen und im Rheinland 1890-1923), 1949, S. 142 ff.

2 Wehler, Die Polen im Ruhrgebiet bis 1918, in: ders. (Hrsg.), Moderne deutsche Sozialgeschichte, 1968, S. 437 ff.

3 Herbert, Geschichte der Ausländerbeschäftigung (Fußn. 1), S. 16. 
den Ruf nach polnischen Landarbeitern durchhalten. Auch die sich ausbreitende illegale Einwanderung hat zu einer im November und Dezember 1890 eingeführten Kompromisslösung geführt. Demnach wurde die Beschäftigung polnischer Arbeiter zugelassen, allerdings ausschließlich von Unverheirateten, um die Sesshaftmachung von Familien zu verhindern, und für eine Karenzzeit vom 1. April bis zum 15. November (die Zulassungszeiten wurden später bis 20. Dezember verlängert). Die Karenzzeit betraf lediglich polnische Landarbeiter. Deutsche Wanderarbeiter wie auch Angehörige anderer Nationen konnten in den Wintermonaten weiter beschäftigt werden. Diese Maßnahme hatte grundsätzlich einen nationalpolitischen Hintergrund. Im preußischen Innenministerium wurde ausdrücklich festgestellt, die Zwangsrückführung der polnischen Arbeiter sei „das einzige Mittel, den ausländischen Arbeitern und auch der heimischen Bevölkerung immer wieder zum Bewusstsein zu bringen, dass sie nur geduldete Fremdlinge seien und ihre dauerhafte Sesshaftmachung ausgeschlossen sei. Dadurch allein könne es vermieden werden, dass die ausländisch-polnischen Arbeiter zu einer Eheschließung mit einer deutschen Staatsangehörigen und Gründung einer Familie schritten, die natürlich dann der Ausweisung ebenso anheimfalle, wie der Ehegatte und der Vater"4. Nach Angaben von U. Herbert machten 1906 die ständig in Preußen lebenden Auslandspolen $7 \%$ der jährlichen polnischen Zuwanderer aus, bei den Nichtpolen hingegen 53\%. 9 von 10 Polen verließen regelmäßig im Spätherbst das preußische Gebiet, um im Frühjahr zurückzukehren. ${ }^{5}$ Diese Maßnahme hat die polnischen Landarbeiter sehr betroffen und die Hoffnungen, die sie mit einer Beschäftigung in Deutschland verbunden hatten, zerstört. Das verdiente Geld wurde nämlich in den Wintermonaten konsumiert und nur selten gelang es, einen Teil des schwerverdienten Geldes zu investieren. ${ }^{6}$

Um eine Kontrolle über die Beschäftigung ausländischer Arbeiter in Deutschland zu erreichen, wurde die „Zentralstelle zur Beschaffung der deutschen Aussiedler und Feldarbeiter“, 1905 in die „Deutsche Feldarbeiterzentrale“ und später in die „Arbeiterzentrale“ in Berlin umgestaltet, errichtet. 1907 wurde der Inlandlegitimierungszwang für Polen eingeführt. 1908 wurde er auf alle ausländischen Arbeiter erweitert. Alle in Deutschland beschäftigten Arbeiter mussten deutsche Inlandspapiere in Form von Legitimationskarten mit Angaben der Personalien des Arbeiters und des Arbeitgebers erhalten. Zur Ausstellung der Legitimationskarten waren die Polizeibehörden unter Mitwirkung der deutschen Arbeiterzentrale berufen. Nach einer weiteren Reform der Ausländerbeschaffung erhielten ausländische Arbeiter die Arbeitskarte bei den Grenzbehörden gegen Gebühr. Für Arbeiter der einzelnen Nationalitäten wurden Arbeitskarten in unterschiedlichen Farben ausgefertigt, für polnische Arbeiter war das eine rote Arbeitskarte.

4 Herbert, Geschichte der Ausländerbeschäftigung (Fußn. 1), S. 24; Bade, Politik und Ökonomie der Ausländerbeschäftigung im preußischen Osten 1885-1914. Die Internationalisierung des Arbeitsmarktes im „Rahmen der preußischen Abwehrpolitik”, in: Puhle/Wehler (Hrsg.), Preußen im Rückblick, 1980, S. $280 \mathrm{ff}$.

5 Herbert, Geschichte der Ausländerbeschäftigung (Fußn. 1), S. 27.

6 Okołowicz, Wychodźtwo i osadnictwo polskie przed wojną światową (Polnische Auswanderung und Ansiedlung vor dem Weltkrieg), 1920, S. 279. 
Die Arbeitskarte war beim Arbeitsantritt dem Unternehmer (der dem Arbeiter nicht bekannt war und an den er für die Saison gebunden war) auszuhändigen. Ein Wechsel der Beschäftigung war nur mit Einwilligung des Arbeitgebers möglich, ein Wechsel ohne dessen Zustimmung galt als Kontraktbruch, ähnlich wie mangelnder Arbeitseifer, und führte zur Kündigung und Ausweisung. ${ }^{7}$

Die Zentralisierung der Ausländerbeschäftigung führte zur Ersetzung der gewerbsmäßig tätigen Arbeitsvermittler durch errichtete Grenzämter.

\section{Rechtliche Grundlagen der Gestaltung der Arbeits- und Lebensbedingungen polnischer Landarbeiter}

Die Rechtsverhältnisse der landwirtschaftlichen Arbeiter und des Gesindes wurden von den reichsgesetzlichen Regelungen, insbesondere der Gewerbeordnung und des BGB ausgeschlossen und dem Landesrecht vorbehalten. Die Rechtsvorschriften für Landarbeiter in Deutschland waren vielschichtig. Es lässt sich „eine eigentümliche Mischung wenn nicht widerstreitender, so doch verschiedenartiger Traditionen beobachten, ein Mit- und Gegeneinander von „,modernem“ Vertragsdenken und „feudal“ bestimmter patriarchalischer Verfügungsmacht. Das ländliche Arbeitsrecht war, darin dem Heereswesen verwandt, eine der Bastionen, von denen aus sich der agrarisch geprägte vorkonstitutionelle Obrigkeitsstaat am beharrlichsten gegen die Herausforderungen des Liberalismus und den Einfluss der heraufziehenden Industriegesellschaft zu verteidigen suchte“. 8 Mit der Aufhebung der Erbuntertänigkeit (1807) beruhte das Verhältnis von Dienstpersonal und Herrschaft auf dem freien Vertrag, dessen Einzelheiten die im November 1810 erlassene „Gesindeordnung für sämtliche Provinzen der preußischen Monarchie“, die an die Stelle der einschlägigen Paragraphen des Allgemeinen Landrechts trat, regelte. Novellierungsversuche der Gesindeordnung, anders als es bei der Gewerbeordnung der Fall gewesen war, scheiterten. In Preußen galten verschiedene Gesindeordnungen, deren Zahl etwa 60 betrug und deren Ursprung bis in das 18. Jahrhundert zurückging. Einzelne ihrer Bestimmungen sind durch das BGB ausdrücklich außer Kraft gesetzt worden (das Züchtigungsrecht der Arbeitgeber), andere als nicht anwendbar erklärt, weil sie gegen den Geist des Reichsrechts verstießen. ${ }^{9}$ Das preußische Gesinderecht war unübersichtlicher denn je, auch deshalb, weil in den annektierten Provinzen eigene Regelungen galten, die durch die Regelungen in den altpreußischen Gebiets-

7 Herbert, Geschichte der Ausländerbeschäftigung (Fußn. 1), S. 34 ff.

8 Flemming, Obrigkeitsstaat, Koalitionsrecht und Landarbeiterschaft. Zur Entwicklung des ländlichen Arbeitsrechts in Preußen zwischen Vormärz und Reichsgründung, in: Puhle/Wehler (Hrsg.), Preußen im Rückblick, 1980, S. 255.

9 Potthoff, Probleme des Arbeitsrechts. Rechtspolitische Betrachtungen eines Volkswirtes, 1912, S. 19. 
teilen nicht automatisch aufgehoben wurden. Ein einheitliches ländliches Arbeitsrecht $\mathrm{zu}$ schaffen, ist der preußischen Staatsverwaltung nicht gelungen. ${ }^{10}$

Wie schon erwähnt, führten gewerbsmäßige Arbeitsvermittler die Rekrutierung nach Deutschland durch. Das Verhältnis zwischen dem Anwerber, der nach der Anwerbung üblicherweise als Vorarbeiter (Vorschnitter) tätig wurde, und dem Landesbesitzer wurde vertraglich geregelt. Es gab aber keine einheitlichen Schnitter-Verträge, ihr Inhalt war nicht eindeutig und so entstanden in wichtigen Angelegenheiten Fragen, die unterschiedlich beantwortet wurden. Hauptsächlich handelte es sich um die rechtliche Position des Vorschnitters in seinem Verhältnis zu dem angeworbenen Arbeiter, wie auch zu dem Arbeitgeber (Grundbesitzer). Strittig war insbesondere die Frage, ob die Schnitter in ein direktes obligatorisches Vertragsverhältnis zu ihrem Arbeitgeber traten, so dass dieser von ihnen unmittelbar die Erfüllung des Arbeitsvertrages verlangen konnte, oder ob ein solches Vertragsverhältnis nur zwischen dem Arbeitgeber und dem Vorschnitter einerseits, dem Vorschnitter und den Schnittern andererseits bestand. ${ }^{11}$ Die Entscheidung in eine dieser Richtungen hatte gravierende Folgen. Einige richterliche Entscheidungen wiesen sind in die Richtung, dass der Vorschnitter, den man entlassen musste oder der freiwillig gegangen war, befugt war, die Schnitter mitzunehmen. Die Position des Vorschnitters war also stark. Was sein Verhältnis zu den Schnittern (polnischen Landarbeitern) betrifft, so berichten die Zeitgenossen, dass Vorschnitter ihre Stelle als Mittel zum leichten, schnellen und oft nicht einwandfreien Geldverdienen benutzten. Es war umso einfacher, da sie meist die Landarbeiter selbst zu entlohnen hatten und Kantinen führten, wo die Arbeiter sich mit Lebensmitteln versorgten, mitunter auch dazu gezwungen waren. Häufig schlossen die Vorschnitter mit den polnischen Landarbeitern einen zweiten polnischen Vertrag, in dem alle Tagelohn- und Akkordsätze niedriger angesetzt wurden. ${ }^{12}$

Die in Annoncen bzw. in Verträgen vorgesehenen Arbeits- und Lohnbedingungen wurden später oft zum Nachteil der Arbeiter geändert oder auch nicht eingehalten, was zu dem sog. Kontraktbruch der polnischen Landarbeiter führte. Übrigens war der Kontraktbruch keine Besonderheit der polnischen Landarbeiter. Die Kontraktbrüche fanden in Deutschland schon viel früher statt und hatten bei verschiedenen Arbeitern wie beim Gesinde, bei Schweizern, Hofgängern und namentlich bei den Landarbeitern ihr besonderes Gepräge. Aufgrund empirischer Forschungen hat der bereits zitierte R. Ehrenberg die Ursachen von Kontraktbrüchen beschrieben. ${ }^{13}$ Beim Gesinde war der Kontraktbruch eine ganz übliche Erscheinung, Tagelöhner und Deputatisten brachen nur selten ihren Kontrakt, bei ledigen Schweizern war der Kontraktbruch sehr üblich, „ebenso schlimm“

10 Flemming, Obrigkeitsstaat (Fußn. 8), S. 267.

11 Ehrenberg/Gehrke, Der Kontraktbruch der Landarbeiter als Massenerscheinung, in: Ehrenberg (Hrsg.), Landarbeit und Kleinbesitz, Heft 1, 1907, S. 14.

12 Ehrenberg/Gehrke, Der Kontraktbruch (Fußn. 11), S. 28, 31.

13 Ehrenberg/Gehrke, Der Kontraktbruch (Fußn. 11); eine umfangreiche Darstellung der Problematik des Kontraktbruches bietet auch Dietz, Vertragsbruch im Arbeits- und Dienstrecht, 1890. 
war er bei Hofgängern. Der Grund für einen Kontraktbruch war überwiegend die Suche nach besseren Arbeitsbedingungen. Die Lage auf dem Arbeitsmarkt, gekennzeichnet durch einen Mangel an Arbeitskräften, begünstigte dieses Verhalten.

Was die Wanderarbeiter (Schnitter), von denen die meisten polnische Landarbeiter waren, anbelangt, so lag die Ursache der Kontraktbrüche bei der Nichteinhaltung der versprochenen Lohn- und Lebensbedingungen, was zur Suche nach besseren Arbeitsbedingungen führte, aber auch bei Abwerbungen durch Bekannte oder sogar im Auftrag anderer Arbeitgeber tätige Personen zu einem anderen Arbeitsplatz, wie auch bei der schlechten Behandlung durch den Arbeitgeber. ${ }^{14}$ Das preußische Landministerium gestand selbst, „dass Kontraktbruch von den ausländischen Arbeitern ziemlich allgemein als die gegebene Selbsthilfe und das einzige [...] Mittel gegen schlechte Behandlung und Verkürzung seitens der Arbeitgeber angesehen und als Ultima Ratio auch von besseren Elementen von vornherein in Ansicht genommen wird.[...]". ${ }^{15}$

Den Angeworbenen wurde ein Mustervertrag, in dem die wichtigsten Arbeits- und Lebensbedingungen aufgezählt wurden, zur Unterzeichnung vorgelegt.

Ein Mustervertrag aus dem Jahre 1913 für polnische Landarbeiter, die für die Provinz Mecklenburg-Schwerin angeworben wurden ${ }^{16}$, enthielt u.a. folgende Bestimmungen: Der $\S 1$ enthielt die Verpflichtung des Arbeiters, jede durch den Arbeitgeber (Stellvertreter) zugeteilte Arbeit sorgfältig und fleißig zu verrichten und den Befehlen des Gutsbesitzers zu folgen. Die Arbeitszeit sollte von 6 Uhr morgens bis 8 Uhr abends mit Frühstücks-, Mittags- und Vesperpausen dauern. Über Überstunden sollte der Arbeitgeber selbständig entscheiden. Im Mustervertrag wurde die pro Woche zustehende Kost in Kartoffeln, Milch und Roggenmehl aufgezählt; die Rubriken betreffend den Tagesbzw. Akkordlohn sollten bei Unterzeichnung des Vertrags unterschrieben werden. Weitere Bestimmungen hatten die Unterbringung der Arbeiter zum Gegenstand. Sie sollte in Sammelräumen stattfinden, jedoch separat für Frauen und Männer, bei Bereitstellung einer Stelle für das Kochen und Waschen. Das Kochen und die Wäschereinigung sollte

14 Bei angespannten Verhältnissen konnte es auch zum „Streik” kommen. Ehrenberg zitiert den Bericht eines Landwirtes: „Im Sommer 1906 streikten alle Schnitter (Russisch-Polen) und legten die Arbeit nieder. Die Ursache war keine Lohnstreitigkeit, sondern der einem Arbeiter gegenüber ausgesprochene Tadel", Ehrenberg/Gehrke, Der Kontraktbruch (Fußn. 11), S. 10.

15 Herbert, Geschichte der Ausländerbeschäftigung (Fußn. 1), S. 42 zitiert eine Stellungnahme zu den Ursachen des Kontraktbruchs des Kommandeurs der mecklenburgisch-schwerinschen Landesgendarmerie von Wettzien. Als häufigste Missstände bei der Behandlung ausländischer Arbeiter werden folgende aufgezählt: „a) Es sind den Arbeitern körperliche Mißhandlungen durch den Dienstherrn oder dessen Beamten, wozu in erster Linie auch Vorschnitter zu rechnen sind, zugefügt worden. b) Es ist der Lohn nicht oder nicht rechtzeitig gezahlt worden, oder es sind davon willkürliche, nicht vertragsmäßige Abzüge gemacht. c) Die gelieferten Nahrungsmittel (Kartoffeln) und die Wohnungen sind in hohem Grade minderwertig. d) Es werden von den Arbeitern andere Arbeiten und Mehrarbeit verlangt, als vertragsmäßig ausbedungen oder ihnen bei Anwerbung als solche bezeichnet sind. e) Die Arbeitsverträge sind den Arbeitern nicht genügend bekannt. f) Die Vorschnitter betrügen die Leute bei der Lohnauszahlung, insbesondere durch die teilweise Auszahlung des Lohns in Waren".

16 Auszüge aus dem Mustervertrag bei Okołowicz, Wychodźtwo i osadnictwo (Fußn. 6), S. 276. 
durch eine Arbeiterin, unter Freistellung von gewöhnlichen Landarbeiten in dieser Zeit, erledigt werden, Die Reisekosten sollte der Arbeitgeber unter dem Vorbehalt tragen, dass bei vorzeitiger Entlassung der Arbeiter dem Arbeitgeber die Kosten zurückerstatten würde.

Bei Erkrankung stand dem Arbeiter nur die Kost ohne Entlohnung zu. Falls am Ort eine Krankenkasse vorhanden war, standen dem Arbeiter die Leistungen der Krankenkasse zu, jedoch unter der Bedingung der Einzahlung der Beiträge. Andernfalls sollte der Arbeitgeber unentgeltlich eine ärztliche Betreuung gewährleisten.

Die in diesem Mustervertrag vorgesehenen Arbeitsbedingungen wurden als ein Fortschritt gegenüber den in früheren Verträgen enthaltenen Bedingungen beurteilt. Insbesondere wurde auf die Bestimmung des $\S 9$ des Mustervertrags hingewiesen, der in Streitfällen die Zuständigkeit eines Gerichts vorsah. Vorher waren für solche Fälle Schlichtungsstellen der „Zentralstelle“ zuständig. ${ }^{17}$ Dennoch enthielt dieser Mustervertrag Elemente „feudal“" bestimmter Verfügungsmacht des Grundbesitzers. Insbesondere charakteristisch ist die Verpflichtung des Arbeiters, Befehle (auf Polnisch: ,rozkazy“) des Gutsbesitzers zu beachten, also die Verwendung einer Terminologie, die im Gesetz betreffend die Verletzungen der Dienstpflichten des Gesindes und der ländlichen Arbeiter vom 24. April 1854 verwendet wurde. 18

Dem Verfasser dieses Aufsatzes sind keine mit deutschen Landarbeitern bzw. mit Landarbeitern anderer Nationalitäten abgeschlossenen Arbeitsverträge zugänglich gewesen. Es ist jedoch ein aus der gleichen Zeit stammender Mustervertrag bekannt, den polnische Landarbeiter mit französischen Arbeitgebern abgeschlossen haben. ${ }^{19}$ Mit allen hier notwendigen Vorbehalten, u.a. bezüglich der Zahl der in Frankreich beschäftigten polnischen Landarbeiter (ca. 10.000 jährlich) und einer anderen Organisation der Beschäftigung, scheint ein vergleichender Blick auf diesen Mustervertrag interessant zu sein.

Besonders bemerkenswert ist, dass in diesem Vertrag in Bezug auf den Landarbeiter ausdrücklich der Begriff „Rechte“ verwendet wurde. In $\S 13$ wurden Gründe einer fristlosen Auflösung des Kontrakts durch den Arbeitgeber aufgezählt (mangelnde Folgsamkeit trotz zweimaliger Ermahnung, Beleidigung des Arbeitgebers oder seines Vertreters, Schwangerschaft einer unverheirateten Arbeiterin, Tierquälerei) und das R e c h t des Arbeiters auf Auflösung des Vertrags: 1) falls der Arbeitgeber den Arbeiter beleidigt und 2) bei Nichteinhaltung der vertraglich festgelegten Arbeitsbedingungen durch den Arbeitgeber. Im letztgenannten Falle sollte der Arbeiter unverzüglich den Polnischen Verein für Emigration benachrichtigen, der intervenieren bzw. an einer Verhandlung vor dem Friedensrichter teilnehmen konnte. Was die Entlohnung betrifft, so wurde im Vertrag ausdrücklich eine Gleichstellung der polnischen Arbeiter mit den einheimischen

17 Okołowicz, Wychodźtwo i osadnictwo (Fußn. 6).

18 Das Gesetz galt noch bis Ende des 19 Jahrhunderts: Dietz, Vertragsbruch (Fußn. 13), S. 8.

19 Okołowicz, Wychodźtwo i osadnictwo (Fußn. 6), S. 281 ff. 
Arbeitern bei Akkordarbeiten, wie auch eine Gleichstellung mit den Einheimischen im Bereich der Verpflegung festgelegt.

Was die tatsächlichen Arbeits- und Lebensbedingungen der polnischen Arbeiter in Deutschland in dieser Zeit anbelangt, so ist eine Differenzierung notwendig. Nach Einschätzungen von J. Okołowicz war die Lage der polnischen Arbeiter in südlichen Teilen Deutschlands wie Bayern oder Württemberg günstiger als in Preußen. Es handelte sich dabei nicht um die Löhne, die vergleichbar waren, sondern um das Verhalten gegenüber den polnischen Arbeitern, das dort viel besser war und die nationalen Vorurteile bis zur Feindlichkeit nicht so spürbar werden ließ. Außerdem galten in den südlichen Ländern der Legitimierungszwang und die Karenzzeit nicht. Darüber hinaus gab es in diesen Ländern im Falle der Landarbeiter aufgrund der Art ihrer Beschäftigung (sie waren in der Regel einzeln bei den Bauern untergebracht) keine Schicht von Aufsehern, die in Preußen zum großen Teil für Misshandlungen und Ausbeutung der polnischen Landarbeiter verantwortlich waren. Bezüglich der Lage der polnischen Landarbeiter in Preußen stellt auch $U$. Herbert fest, dass die Arbeits- und Lebensbedingungen der Landarbeiter schlecht waren. ${ }^{20}$ Dies lag jedoch nicht allein an der nationalistisch begründeten Polenfeindlichkeit, sondern eher an der Struktur des Beschäftigungsverhältnisses. Um überhaupt Arbeiter zu bekommen, wurden den Polen relativ hohe Löhne versprochen und bei der Anwerbung vereinbart. Am Ende der Saison erhielten die Polen oft viel weniger. „Da es eine organisierte Interessenvertretung der Zuwanderer nicht gab, die Polen meist kein Deutsch konnten und Beschwerden oder Lohnforderungen als „Unbotmäßigkeit“ zur Entlastung und Abschiebung führen konnten, gab es gegen die systematischen Übervorteilungen durch die landwirtschaftlichen Arbeitgeber kein hemmendes Gegengewicht".21

Was die Lage der polnischen Arbeiter im Bereich der Sozialversicherung betrifft, so umfasste die Invalidenversicherung nur einen Teil der Arbeiter, nämlich nur die Arbeiter, die in Ländern des Deutschen Reiches beschäftigt waren, wo keine Karenzzeit eingeführt wurde. In Ländern mit Karenzzeit wurden die polnischen Landarbeiter gemäß einer auf $\S 1233$ der RVO gestützten Bekanntmachung des Vereinsrats vom 7. März 1901 (Rada Związkowa) von der Invalidenversicherung befreit. Nach § 1233 konnte die Reichsregierung bestimmen, dass Ausländer versicherungsfrei waren, denen die Behörde den Aufenthalt im Inland nur für eine bestimmte Dauer gestattet hatte. Die Arbeitgeber zahlten dann soviel an die Landesversicherungsanstalt, wie sie sonst aus eigenen Mitteln zahlen müssten. Die Unfallversicherung umfasste zwar die polnischen Landarbeiter, jedoch konnten sie die Leistungen dieser Versicherung wegen der Vorschriften der RVO über das Ruhen der Ansprüche beim Auslandsaufenthalt der Berechtigten Ausländer (§§ 203.1, 586, 614) praktisch nicht in Anspruch nehmen.

20 Herbert, Geschichte der Ausländerbeschäftigung (Fußn. 1), S. 42.

21 Herbert, Geschichte der Ausländerbeschäftigung (Fußn. 1), S. 42. 


\section{Die rechtliche Lage der polnischen Landarbeiter während des 1. Weltkrieges}

Die Zeit des 1. Weltkrieges verdient besondere Aufmerksamkeit. Kurz nach Kriegsausbruch ordnete das Preußische Kriegsministerium an, dass die Landarbeiter aus dem feindlichen Ausland, die sich auf dem Gebiet des Deutschen Reichs aufhielten, d.h. vor allem die russisch-polnischen Saisonarbeiter, daran zu hindern seien, in ihre Heimatländer zurückzukehren. Diese Anordnung wurde auch auf die in der Industrie Beschäftigten ausgeweitet, so dass im Endeffekt ca. 300.000 der sog. russisch-polnischen Arbeiter gezwungen waren, weiterhin in Deutschland zu verbleiben. Die Rückkehr wurde aus militärischen Gründen ohne Zeitbegrenzung verboten. Aus einem „Rückkehrzwang“ entstand also ein „Rückkehrverbot“. ${ }^{22}$ Die alte Arbeitsstelle durfte nicht selbständig verlassen werden. Eine allgemeine rechtliche Grundlage für eine Arbeitsverpflichtung wurde aus Rücksicht auf das Völkerrecht wie auch im Hinblick auf mögliche Reaktionen des Auslands nicht erlassen. In der Praxis galten die Befehle der Generalkommandos mit Androhung von Haftstrafen für die Niederlegung oder Verweigerung der Arbeit wie auch für den Verstoß gegen andere Maßnahmen, hier z.B. Quartier- und Ausgangssperre.

Rückkehr- und Ortswechselverbot sowie die Bindung an den Arbeitgeber verbunden mit den Strafandrohungen wirkten sich negativ auf andere Arbeitsbedingungen, insbesondere auf die Höhe der Löhne, aus, die durch die Arbeitgeber gedrückt wurden. All das verursachte einerseits noch strengere Maßnahmen, andererseits hatte es negative Auswirkungen auf die Arbeitsleistung ausländischer Arbeiter.

1917 entschied man sich für einige Erleichterungen. Man ließ die Möglichkeit zu, den Ort und die Arbeit zu wechseln, es wurden Schlichtungsstellen einberufen und die Bestimmungen zu Urlaubsheimfahrten gelockert. Gemäß dem Erlass vom 15. Oktober 1917 sollte jedem polnischen Arbeiter jährlich eine Urlaubsheimreise gewährt werden, die Arbeits- und Lebensbedingungen sollten durch Fürsorgekommissionen überprüft und Missstände abgestellt werden. Die Verhältnisse auf dem Lande änderten sich jedoch bis Kriegsende nur wenig. ${ }^{23}$

Im deutschen historischen Schrifttum wird die Beschäftigung polnischer Arbeiter in Deutschland während des 1. Weltkrieges als eine Art von Zwangsarbeit qualifiziert. Die Arbeiter unterlagen nämlich besonderen Rechtsvorschriften, man brachte sie in Lagern unter, sie mussten Beschränkungen im Bereich der Freizügigkeit einhalten, waren bei Arbeitsverweigerung inhaftiert und oft Misshandlungen sowie Beleidigungen ausgesetzt. $^{24}$

22 Umfangreich dazu Herbert, Geschichte der Ausländerbeschäftigung (Fußn. 1), S. 82 ff.

23 Herbert, Geschichte der Ausländerbeschäftigung (Fußn. 1), S. 82 ff.

24 Herbert, Geschichte der Ausländerbeschäftigung (Fußn. 1), S. 82 ff. 


\section{Die Ruhrpolen in den Industriegebieten Preußens}

Wie schon erwähnt wurde, sind die sog. Ruhrpolen aus den östlichen Provinzen Preußens in das Ruhrgebiet mit der Absicht eines längeren Aufenthalts, des Aufbaus einer neuen Existenz und oft mit ihren Familien angekommen. Die Eingewöhnung wurde aber von Spannungen und Anpassungsschwierigkeiten begleitet. Sie konnten zwar anfangs unterschiedliche Vereine gründen, kirchliche, sportliche, auch politische, bis hin zur Gründung von Gewerkschaftsorganisationen. ${ }^{25}$ Diese Vereine waren wegen ihrer sozialpsychologischen Funktion von großer Bedeutung. Im Laufe der Zeit haben sich die Nationalitätenkonflikte im preußisch-deutschen Reich der Bülowzeit aber zugespitzt, was in einigen die Polen diskriminierenden Rechtsakten resultierte. Als Zäsur wird in dieser Hinsicht auf die Bergpolizeiverordnung des königlichen Oberbergamtes Dortmund hingewiesen. ${ }^{26}$ In dieser Verordnung wurde festgesetzt, dass fremdsprachige Arbeiter nur dann in den Bergwerken beschäftigt werden sollten, „wenn sie genügend deutsch verstehen um mündliche Anweisungen ihrer Vorgesetzten und Mitteilungen ihrer Mitarbeiter richtig aufzufassen“. Alle gehobenen Stellungen waren Fremdsprachigen nur unter der Bedingung zugänglich, dass sie Deutsch sprachen und in Schrift und Druck lesen konnten ( $(2)$. Nach der Einschätzung von H. U. Wehler sollen allein sachliche Gesichtspunkte für den Erlass der Verordnung maßgebend gewesen sein; so habe es im Interesse der fremdsprachigen Arbeiter, auch der deutschen Bergleute und der Zechenverwaltungen gelegen, zumindest die wichtigsten Sicherheitsvorschriften in Übersetzung bekanntzumachen, wie das im Oberbergamtsbezirk Breslau der Fall war. ${ }^{27}$ Eine weitere Maßnahme war das Reichsvereinsgesetz (1908). Gemäß $§ 12$ dieses Gesetzes war in allen öffentlichen Versammlungen Polnisch als Verhandlungssprache untersagt. Im Ergebnis durfte im Ruhrgebiet in öffentlichen Versammlungen nicht mehr polnisch gesprochen werden. Diese Vorschrift war ein schweres Hemmnis für das verzweigte polnische Vereinswesen, sie hat umgekehrt wesentlich dazu beigetragen, das Solidaritätsgefühl der Polen zu stärken, die nationalen Gegensätze zu verschärfen und den Assimilierungsprozess zu verzögern. ${ }^{28}$

25 Wehler, Die Polen im Ruhrgebiet (Fußn. 2), S. 445; Murzynowska, Polskie wychodźtwo zarobkowe w Zagłębiu Ruhry w latach 1880-1914 (Die polnische Auswanderung im Ruhrgebiet in den Jahren 1880-1914), 1972, S. 92 ff., S. 241 ff.; s. auch Pallaske (Hrsg.), Die Migration von Polen nach Deutschland. Zu Geschichte und Gegenwart eines europäischen Migrationssystems, 2001.

26 Wehler, Die Polen im Ruhrgebiet (Fußn. 2), S. 452; s. auch Herbert, Geschichte der Ausländerbeschäftigung (Fußn. 1), S. 71.

27 Wehler, Die Polen im Ruhrgebiet (Fußn. 2), S. 453.

28 Wehler, Die Polen im Ruhgebiet (Fußn. 2), S. 455; s. auch Kleßmann, Polnische Bergarbeiter im Ruhrgebiet 1870-1945, 1975, insbes. S. 73 ff. 


\section{B. Die rechtliche Lage der polnischen Arbeiter in der Weimarer Republik}

\section{Vorbemerkungen}

Nach dem 1. Weltkrieg wurden im Rahmen der Demobilmachung zunächst die Grenzen für die zuwanderwilligen Ausländer gesperrt und eine Zurückführung der sich in Deutschland befindlichen Arbeitnehmer organisiert. In einer relativ kurzen Zeit gelang es, die Zahl der ausländischen Arbeiter in Deutschland bis 1924 von ca. 2 Mio. (davon ca. 1 Mio. Kriegsgefangene) auf 174.000 zu reduzieren. ${ }^{29}$ Von den sich im Lande befindlichen polnischen Arbeitern ist ein Teil nach Frankreich gegangen, ein Teil hat eine Beschäftigung in der oberschlesischen Industrie gefunden, der restliche Teil, überwiegend Landarbeiter, ist in Deutschland geblieben. Trotz des Zurückführungsprozesses hat der Druck der landwirtschaftlichen Interessengruppen bewirkt, dass eine Genehmigung für die Beschäftigung von etwa 50.000 polnischen Landarbeitern in Ostdeutschland erteilt wurde.

Die Beziehungen zwischen Deutschland und Polen nach dem 1. Weltkrieg waren gespannt. Noch ehe die neue Regelung deutsch-polnischer Grenzen in Kraft trat, deutete sich der Wechsel bereits an, der die Zeit der Weimarer Republik bezüglich der zwischenstaatlichen Verhältnisse charakterisierte. Außer diplomatischen gab es auch kämpferische Auseinandersetzungen, wie es der Fall in Oberschlesien war. Es wurde auch mit ökonomischen Mitteln gekämpft (der sog. Zollkrieg). Dennoch zeigten beide Staaten Bereitschaft zur Lösung der durch territoriale Verschiebungen entstandenen Probleme. Stufenweise und nicht ohne Schwierigkeiten wurden die durch territoriale Verschiebungen entstandenen Verrechnungsprobleme zwischen den deutschen und polnischen Sozialversicherungsträgern durch bilaterale Abkommen geregelt. ${ }^{30}$ Die territorialen Verschiebungen bewirkten auch, dass ehemalige deutsche Bürger polnische Bürger wurden. In diesem Zusammenhang und in Verbindung mit dem Territorialitätsprinzip der Reichsversicherungsordnung im Bereich der Leistungserbringung entstanden gewichtige soziale Probleme, die einer Lösung bedurften. Darüber hinaus ging es aber auch um die Regelung einiger Probleme der polnischen Arbeiter im Bereich der sozialen Sicherheit, die aus der Zeit vor 1918 stammten. Nicht weniger wichtig waren die Beschäftigungsbedingungen polnischer Arbeiter während der Zeit der Weimarer Republik.

Relativ günstige Voraussetzungen für eine Normalisierung der deutsch-polnischen Verhältnisse ergaben sich seit 1926/1927. In den Jahren von 1927 bis 1931 konnten

29 Herbert, Geschichte der Ausländerbeschäftigung (Fußn. 1), S. 114.

30 S. insbesondere das in Genf abgeschlossene deutsch-polnische Abkommen über Oberschlesien vom 11. u. 12. Juni 1922, RGB1. II, 1922, S. 237, 585; s. auch die Verordnung über die Rechtsverhältnisse aufgelöster Versicherungsträger in den damaligen Provinzen Westpreußen und Posen vom 28. März 1923, RGBl. I, 1923, S. 221. 
wichtige Abkommen, an welchen übrigens schon seit einigen Jahren gearbeitet worden war, unterzeichnet werden.

Auch einige allgemeine Faktoren beeinflussten die Entwicklung der rechtlichen Regulierungen. Dazu ist der sich ausbreitende Gedanke des Sozialstaates zu rechnen, der in der Weimarer Reichsverfassung Ausdruck fand. ${ }^{31}$ Ein weiterer Faktor war das neue internationale Umfeld, das durch das Entstehen und die Tätigkeit der Internationalen Arbeitsorganisation aufgebaut wurde und auf das Handeln der Mitgliedstaaten einwirkte. Art. 162 der Weimarer Verfassung bestimmte, dass Deutschland auf die internationale Standardisierung der Arbeits- und Sozialgesetzgebung hinwirken sollte. Der Einfluss der IAO war insbesondere im Bereich der Gestaltung der Verhältnisse der sozialen Sicherheit ausländischer Arbeitnehmer sichtbar.

\section{Die arbeitsrechtliche Lage der polnischen Landarbeiter}

Im Sommer 1918 wurde auf Veranlassung des Kriegsausschusses der deutschen Landwirtschaft ein Entwurf einer einheitlichen Landarbeitsverordnung ausgearbeitet, aus welchem die vorläufige Landarbeitsordnung vom 24. Januar 191932 hervorging. Im Rahmen der Revolutionsverordnungen wurden die Gesindeordnungen aufgehoben. ${ }^{33}$ Für das Vertragsrecht in der Land- und Forstwirtschaft wurden das allgemeine BGB und diese Landarbeitsordnung maßgebend.

Für ausländische Landarbeiter galten neben der vorläufigen Landarbeitsordnung besondere Vorschriften, nämlich die Verordnung über die Anwerbung und Vermittlung ausländischer Landarbeiter vom 19. Oktober 1922 und die Verordnung über die Einstellung und Beschäftigung ausländischer Arbeiter vom 2. Januar $1926^{34}$, wie auch ein auf diese Vorschriften gestützter Vertrag, eine Art von Mustervertrag. Gemäß $§ 4$ der erstgenannten Verordnung durften ausländische landwirtschaftliche Wanderarbeiter nur auf Grund des vom landwirtschaftlichen Fachausschuss der Reichsanstalt für Arbeitsvermittlung und Arbeitslosenversicherung (Hauptstelle) aufgestellten Arbeitsvertrages für ausländische Wanderarbeiter angeworben und vermittelt werden. Andere Arbeitsverträge mit diesen Arbeitern waren insoweit unwirksam, als sie von dem genannten Arbeitsvertrag zu Ungunsten der Arbeitnehmer abwichen. An Stelle der unwirksamen Vereinbarungen traten die entsprechenden Bestimmungen des Musterarbeitsvertrags.

31 Zacher, Aktuelle Herausforderungen für die Sozialversicherung, in: Eichenhofer (Hrsg.), Bismarck, die Sozialversicherung und deren Zukunft, 2000, S. 33 ff. und S. 81 ff.; s. auch Eichenhofer, Internationales Sozialrecht, 1994, S. 33.

32 RGBl. I, 1919, S. 111 mit mehreren Änderungen; näher dazu Molitor, Kommentar zur vorläufigen Landarbeitsordnung vom 24. Januar 1919, 1928, S. 2 ff.

33 Hueck/Nipperdey, Lehrbuch des Arbeitsrechts, Bd. I, 1931, S. 22.

34 RGBl. I, 1926, S. 5. 
Für die Anwerbung, Vermittlung und „Verpflichtung” zur Beschäftigung waren ausschließlich die staatlichen Arbeitsvermittlungsämter zuständig. Der Anwerbung der ausländischen Arbeiter ging ein Vertrag der deutschen Regierung und der Regierung des Herkunftslandes voraus. Ein entsprechender Vertrag mit der polnischen Regierung wurde am 24. November 1927 abgeschlossen. Die Reichsanstalt für AVAV entsandte ihre mit Aufträgen der Landesarbeitsämter ausgestatteten Werber in die Vertragsstaaten, wo sie den von ihnen gewählten Landarbeitern die durch die Betriebsleiter unterzeichneten Arbeitsverträge zur Unterschrift vorlegten und damit die ausländischen Arbeiter zur Beschäftigung in der Landwirtschaft verpflichteten.

Der Mustervertrag wurde in zehn umfangreiche Abschnitte aufgeteilt: I. Dauer des Arbeitsverhältnisses, II. Arbeitszeit, III. Löhne, IV. Naturalien und Wohnung, V. Reisekosten, VI. Arbeitsgeräte, VII. Moralisches Verhalten, vorzeitige Lösung des Vertrages, VIII. Erkrankung, Versicherung, IX. Fürsorge und Beilegung von Beschwerden, X. Austragung von Streitigkeiten.

Die Arbeitszeit sollte sich nach den Bestimmungen der Kreis- bzw. Bezirkstarife richten. Für den Fall, dass keine tariflichen Regelungen bestanden, hatte der Vertrag einige allgemeine Grundsätze der Arbeitszeitgestaltung formuliert: Zwei Stunden zur Einnahme von Frühstück, Mittagessen und Vesper sollten in die Arbeitszeit nicht eingerechnet werden, während der Weg vom Hof zur Arbeit und von der Arbeit zum Hof in die Arbeitszeit eingerechnet wurde. Den römisch-katholischen Arbeitnehmern wurde im Vertrag das Recht auf Ruhetage an den dort aufgezählten katholischen Feiertagen zugesprochen.

Im Abschnitt über Löhne wurden folgende im Verhältnis zu der Zeit vor 1918 neue Grundsätze der Entlohnung vorgesehen: Gleichstellung der ausländischen mit den deutschen Wanderarbeitnehmern, falls ein gültiger Tarifvertrag bestand, andernfalls Gleichstellung im Bereich des Gesamtentgelts, das die deutschen Arbeiter in einer entsprechenden Kategorie erhielten.

Im Hinblick auf die gegenüber polnischen Landarbeitern vor 1918 praktizierten Verfahren war folgende Bestimmung des Vertrages von großer Bedeutung: „Das Auslohnen hat nur durch die Arbeitgeber oder deren Beamte im Beisein der Aufseher zu erfolgen, niemals durch den Aufseher, Vorschnitter oder Vorarbeiter allein". Auch die Bestimmung über die Möglichkeit einer Beanstandung der Lohnabrechnung war sehr wichtig und deutete eine neue rechtliche Lage der Landarbeiter an.

Im Hinblick auf die Lage der polnischen Landarbeiter vor 1918 waren ebenso die Bestimmungen des VII. Abschnitts über moralisches Verhalten und vorzeitige Lösung des Vertrages wichtig. Unter anderem enthielt dieser die Bestimmung „Geldstrafen könn[t]en gegen einen Arbeiter nur auf Grund einer vorher erlassenen, an sichtbarer Stelle ausgehängten Arbeitsordnung verhängt werden. Bei Vorliegen eines wichtigen Grundes, insbesondere, wenn einer der Vertragsschließenden gegen den anderen Tätlichkeiten verübt, grobe Beleidigungen ausspricht oder sich unsittlich verhält, ist der andere Vertragsschließende zur sofortigen Lösung des Arbeitsverhältnisses berechtigt. 
Als wichtiger Grund gelten ferner für den Arbeitgeber beharrliche Verweigerung oder grobe Vernachlässigung der Dienstleistung seitens des Arbeitnehmers, für den Arbeitnehmer Versagung des vereinbarten Lohnes und wiederholt unpünktliche Lohnzahlung seitens des Arbeitgebers, sowie anhaltend - d.h. trotz mehrfacher Vorstellungen unverändert - schlechte Kost oder gesundheitsschädliche Wohnung". Zu den Fürsogepflichten des Arbeitgebers gehörte die Bereitstellung von gemeinschaftlichen, einwandfreien und mit Tischen, Sitz- und Waschgelegenheiten ausgestatteten Wohnungen, nach Geschlechtern getrennt, mit gemeinschaftlicher Feuerstelle zum Kochen und Waschen, ausreichendem Heizungsmaterial und außerdem Bettstelle, Strohsack, Kopfkissen und wollener Decke für jede Person. Die Unterkunft sollte hygienischen Vorschriften entsprechen. Die Beachtung der Schutzbestimmungen des Vertrages wurde den Beauftragten der deutschen Arbeiterzentrale in Berlin, die jederzeit das Recht des freien Zutritts zu den Wohn- und Arbeitsstätten der Arbeiter hatten, übertragen. Sie waren auch verpflichtet, Beschwerden der Arbeitgeber und Arbeitnehmer entgegenzunehmen und möglichst an Ort und Stelle beizulegen (Abschnitt IX). Der Zugang zum Arbeitsgericht wurde eröffnet.

Die ausländischen Landarbeiter hatten keinen Anspruch auf Urlaub. Sie mussten auch ein teilweises Einbehalten des Lohnes dulden, der erst bei ordnungsgemäßer Beendigung des Arbeitsverhältnisses vollständig ausbezahlt wurde. Diese Bestimmung sollte die sog. Kontraktbrüche verhindern.

Einige Angelegenheiten der Beschäftigung polnischer Landarbeiter wurden im Vertrag zwischen dem Deutschen Reich und der Republik Polen vom 24. November 1927 über polnische Landarbeiter geregelt. ${ }^{35}$ Die Beschäftigung sollte grundsätzlich den Charakter einer Saisonarbeit haben. Polen, die vor dem 1. Januar 1919 nach Deutschland gekommen und dort geblieben waren, bekamen das Recht, als landwirtschaftliche Arbeiter in Deutschland zu bleiben und für ihre Tätigkeit einen Befreiungsschein zu erhalten. Die in der Zeit zwischen dem 1. Januar 1918 und dem 31. Dezember 1925 nach Deutschland Angekommenen und dort Verbliebenen wurden stufenweise nach festgelegten Jahresraten in die Wanderarbeiterbewegung eingereiht, wobei solche Personen ausgeschlossen werden sollten, für die dies eine besondere Härte bedeuten würde (Art. 3, 4 des Vertrages).

Gemäß Art. 7 des Vertrages sollten die polnischen landwirtschaftlichen Arbeiter in Deutschland hinsichtlich des Arbeitsschutzes, der gewerkschaftlichen Betätigung, der öffentlichen Fürsorge und der Regelung der Arbeitsbedingungen einschließlich des Schlichtungswesens und der Arbeitsgerichtsbarkeit den gleichen Schutz wie die deutschen Arbeiter genießen, jedoch mit dem Vorbehalt, dass nicht die deutsche Gesetzgebung bezogen auf ausländische Staatsangehörige im Allgemeinen etwas anderes bestimmte.

35 RGBl. II, 1928, S. 167. 
In Art. 8 hatte sich die deutsche Regierung verpflichtet, ihren Einfluss dahingehend geltend zu machen, dass die Wohnungen der polnischen landwirtschaftlichen Wanderarbeiter in sittlicher und gesundheitlicher Hinsicht einwandfrei werden sollten.

Was die faktische Lage der polnischen Saisonarbeiter anbelangt, so ergaben die Untersuchungen eines polnischen Soziologen von 1937, dass die meisten Polen nur für die Dauer der Erntezeit als Saisonarbeiter arbeiteten, zwei Drittel von ihnen Frauen waren, Löhne, Unterkunft und Verpflegung zwar nicht gut waren, aber den seit Jahrzehnten üblichen Gepflogenheiten entsprachen. Die Arbeitszeiten waren außerordentlich lang. Insgesamt waren die Verhältnisse der polnischen Landarbeiter in Deutschland nach Einschätzung von $J$. Sobczak, „fast die gleichen, wenigstens nicht schlimmer als die vor 1932 “.36

\section{Gestaltung der sozialversicherungsrechtlichen Verhältnisse}

Dem beabsichtigten allgemeinen Abkommen über die Sozialversicherung ging die Regelung zweier sozialversicherungsrechtlicher Teilbereiche voran. Der erste wurde im Abkommen über die Erwerbslosenfürsorge und Arbeitslosenversicherung vom 14. Juli 1927 geregelt. Der zweite Teilbereich, der zwar alle Gebiete der Sozialversicherung umfasste, sich jedoch lediglich auf Landarbeiter bezog, wurde im zweiten Teil des bereits besprochenen Vertrages zwischen dem Deutschen Reiche und der Polnischen Republik über polnische landwirtschaftliche Arbeiter vom 31. März 1928 geregelt.

In einem der ersten Abkommen der IAO wurde die Gewährung sozialer Sicherheit im Falle der Arbeitslosigkeit geregelt. Mitgliedstaaten, die das Abkommen ratifiziert hatten, wurden verpflichtet entsprechende Vereinbarungen zu treffen, damit den Arbeitnehmern eines anderen Staates im Falle der Arbeitslosigkeit dieselben Leistungen gewährt wurden, wie den eigenen Arbeitern. Polen ratifizierte das Abkommen der IAO am 21. Juni 1924 und Deutschland am 6. Juni 1925. Das bereits erwähnte deutsch-polnische Abkommen aus dem Jahre 1927 war einerseits die Erfüllung dieser Verpflichtung, bezweckte aber darüber hinaus eine Normalisierung der deutsch-polnischen Verhältnisse im Bereich der sozialen Sicherheit.

Die wichtigste Bestimmung des Abkommens von 1927 befindet sich in Art. 1. Gemäß dieser Vorschrift sollten den polnischen Staatsangehörigen im Deutschen Reich die Leistungen der Erwerbslosenfürsorge und nach Einführung einer Arbeitslosenversicherung deren Leistungen unter den gleichen Voraussetzungen und in gleichem Umfang gewährt werden wie den deutschen Reichsangehörigen. Ebenso standen deutschen Reichsangehörigen in der Polnischen Republik die Leistungen der polnischen Arbeitslosenversicherung unter den gleichen Voraussetzungen und im gleichen Umfang zu wie den polnischen Staatsangehörigen. Das gleiche galt für Leistungen der Krisenfürsorge

36 Herbert, Geschichte der Ausländerbeschäftigung (Fußn. 1), S. 121. 
(Art. 2). Es ist zu erwähnen, dass in der Zeit der Unterzeichnung des Abkommens in Polen das Gesetz vom 18. Juli 1924 (novelliert am 28. Oktober 1925) über die Versorgung im Falle der Arbeitslosigkeit galt. ${ }^{37}$ Anders als in Deutschland bedurfte das Abkommen in Polen keiner Änderung der bestehenden Regelungen, weil das Gesetz vom 6. Juli 1923 Ausländer und Inländer im Bereich der sozialen Sicherheit gleichgestellt und den Leistungsexport vorgesehen hatte. ${ }^{38}$

Wichtig war die Bestimmung des Art. 3, wonach die nach den Art. 1 und 2 gewährten Leistungen keine Folgen der öffentlichen Fürsorge (Armenpflege) hatten. Es war nämlich dazu gekommen, dass arbeitslose Polen teilweise auf die Unterstützung des polnischen Konsulats angewiesen waren und teilweise aus Deutschland ausgewiesen wurden, weil sie zu einer Last für die öffentliche Fürsorge geworden waren. ${ }^{39}$

Im Schlussprotokoll erklärten die Parteien einvernehmlich, dass polnische landwirtschaftliche Arbeiter so lange nicht von diesem Abkommen erfasst waren, und demnach auch die Leistungen der deutschen Erwerbslosenfürsorge nicht erhielten, solange sie im Deutschen Reich von der Beitragspflicht zur Erwerbslosenfürsorge befreit waren. Aufgrund ihrer Beschäftigungsart hätten sie eigentlich gar keine Möglichkeit gehabt, diese Leistungen zu bekommen. Bis zu diesem Zeitpunkt waren die polnischen Landarbeiter beitragspflichtig, konnten aber die Leistungen der Erwerbslosenfürsorge nicht in Anspruch nehmen. 40

Insgesamt wurde dieses Abkommen von polnischer Seite aus positiv bewertet. Man sah in ihm einen guten Anfang zur Gestaltung zwischenstaatlicher Sozialversicherungsverhältnisse zwischen Polen und anderen Staaten, in denen Polen beschäftigt waren. ${ }^{41}$

Wie schon erwähnt, enthielten die deutschen Sozialversicherungsvorschriften einige Regelungen, die für ausländische Arbeiter und insbesondere für polnische Landarbeiter ungünstig waren. Die polnische Regierung hat nach 1918 versucht, einige Probleme mittels provisorischer Vereinbarungen zu lösen. Eine Gesamtregelung brachte der teilweise schon besprochene Vertrag über polnische landwirtschaftliche Arbeiter vom 24. November 1927. Die wichtigste Regelung im Bereich der Sozialversicherung befand sich in Art. 11. Danach sollten polnische Landarbeiter und ihre Hinterbliebenen deutschen Staatsangehörigen und ihren Hinterbliebenen in Bezug auf die Leistungen der deutschen Kranken- und Unfallversicherung gleichgestellt werden. Diese allgemeine Regelung bedurfte jedoch einer Ergänzung im Hinblick auf die Merkmale der Beschäf-

37 Dziennik Ustaw Rzeczpospolitej Polskiej (Gesetzblatt der Republik Polen, im Folgenden: Dz. U. R. P.) Nr. 67, Pos. 650.

38 Dz. U. R. P. Nr. 73, Pos. 587.

39 S. Kommentar zum Abkommen „Układ polsko-niemiecki w przedmiocie wzajemnych stosunków w dziedzinie ustawodawstwa społecznego, dotyczącego bezrobocia" (Das polnisch-deutsche Abkommen im Bereich der gegenseitigen Verhältnisse in der Sozialgesetzgebung betr. der Arbeitslosigkeit) in der Zeitschrift „Praca i Opieka Społeczna” (Arbeit und Sozialfürsorge, im Folgenden: Pr. i O. S.) 1931, Nr. 4, S. 81.

40 Pr. i O. S. 1931, Nr. 4 (Fußn. 39), S. 82.

41 Pr. i O. S. 1931, Nr. 4 (Fußn. 39), S. 82. 
tigung polnischer Landarbeiter und die in der Reichsversicherungsordnung enthaltenen Regelungen. Einige Vorschriften der RVO knüpften nämlich an die Bedingung einer häuslichen Gemeinschaft des Berechtigten beim Eintritt des Versicherungsfalles an, die als Voraussetzung für die Leistungen galt. Die Erfüllung dieser Voraussetzung im Falle der polnischen Landarbeiter war praktisch nicht möglich. Deshalb wurde in Art. 12 des Vertrages eine juristische Fiktion eingeführt. Bei der Anwendung des $\S 203$ Nr. 1 und des $\S 614$ der Reichsversicherungsordnung sollte für einen polnischen Landarbeiter, der nicht im Besitz eines Befreiungsscheins war, die häusliche Gemeinschaft, in der er mit seinen Angehörigen in Polen lebte, als nicht unterbrochen gelten, solange sich dieser Arbeiter recht- und vorschriftsmäßig in Deutschland aufhielt. Ebenso wurde in Art. 13 im Hinblick auf die Vorschriften der RVO über Abfindungen von Ansprüchen aus der deutschen Unfallversicherung bestimmt, dass der Aufenthalt in Polen nicht als Aufenthalt im Ausland galt.

Die Invalidenversicherung umfasste, wie schon erwähnt, nur einen Teil der polnischen Arbeiter, nämlich solche mit einem Befreiungsschein und solche, die in Ländern des Reiches beschäftigt waren, in denen sie einen ständigen Aufenthalt hatten (Art. 14). Die meisten der landwirtschaftlichen Arbeiter wurden von der Invalidenversicherung befreit. Gemäß Art. 16 des Vertrages sollten die vertragsschließenden Parteien in Verhandlungen treten, sobald Polen für sein ganzes Gebiet eine Invaliden- und Hinterbliebenenversicherung für landwirtschaftliche Arbeiter geschaffen hatte, ,um durch Übereinkommen dafür zu sorgen, dass auch polnische Wanderarbeiter, die keinen Befreiungsschein erhalten hatten, während ihrer Beschäftigung in Deutschland die Vorteile einer Invalidenversicherung genossen.

Aus polnischer Sicht wurde dieser Vertrag positiv beurteilt. Einige Probleme, die bei der praktischen Realisierung des Vertrages aufgetreten waren, z. B. bezüglich der Auslegung seines persönlichen Anwendungsbereiches, konnten im Einvernehmen der beiden Vertragsseiten gelöst werden. 42

Die größte Bedeutung kam dem dritten in der Reihe der Sozialversicherungsabkommen zu, nämlich dem Vertrag zwischen dem Deutschen Reich und der Republik Polen über die Sozialversicherung vom 11. Juni 1931.43 Seine Bedeutung lag im sachlichen Bereich und in den im Vertrag eingeführten Lösungen. Außerdem war er für den polnischen Haushalt günstig, der bis zu diesem Zeitpunkt aufgrund des Ruhens der Ansprüche der polnischen Arbeiter gegenüber den deutschen Sozialversicherungsträgern an ehemalige ausländische Arbeiter verschiedene Unterstützungsgelder und vorläufige Leistungen gezahlt hatte. Dagegen hatte Polen ohne Gegenleistung deutschen Sozialver-

42 Szymanko, Postanowienia ubezpieczeniowe konwencji polsko-niemieckiej z dnia 24 listopada 1927 o polskich robotnikach rolnych (Die sozialversicherungsrechtlichen Bestimmungen des polnischdeutschen Vertrags vom 24. November 1927 über polnische Landarbeiter), Pr. i O. S. 1931, Nr. 1, S. $32 \mathrm{ff}$.

43 RGB1. II, 1933, S. 645. 
sicherten auch Leistungen ins Ausland gezahlt. Wie schon erwähnt, enthielten Vorschriften der polnischen Sozialversicherung den Grundsatz des Leistungsexportes.

Dieser Vertrag wurde sehr lange ausgehandelt. Die Verhandlungen dauerten insgesamt 10 Jahre, wobei die Unterzeichnung wegen einer beim Völkerbund eingereichten polnischen Klage verschoben wurde. ${ }^{44}$ Der Vertrag ist umfangreich; er zählt 50 Artikel und 13 im Schlussprotokoll enthaltene Bestimmungen. Er stützte sich auf moderne Lösungen, die teilweise denen vorangegangen sind, die in den Übereinkommen der IAO ihren Platz fanden. Neben den im Abkommen aus den frühen Jahren des 20. Jahrhunderts genannten Gewährleistungen ${ }^{45}$, wie lex loci laboris, Leistungsexport und Gewährung sowie Umfang von Sozialleistungen unabhängig von der Staatsangehörigkeit, enthielt dieser Vertrag weitere Grundprinzipien, die erst später in das Abkommen der IAO Nr. 48 von 1935 aufgenommen wurden. Es ist zu erwähnen, dass der Vertrag im Gegensatz zu dem Abkommen, das sich auf die Koordination der Alterssicherung konzentrierte, mehrere Bereiche der Sozialversicherung umfasste.

Der Vertrag bezog sich auf folgende Zweige der Sozialversicherung: 1) die Krankenversicherung, 2) die Unfallversicherung, 3) die Invalidenversicherung, 4) die Angestelltenversicherung und 5) die knappschaftliche Versicherung (Art. 1).

In Art. 2 wurde der Grundsatz lex loci laboris festgeschrieben. Er bestimmte, dass bei der Durchführung der bezeichneten Zweige der Sozialversicherung grundsätzlich die Rechtvorschriften des Staates Anwendung fanden, in dem die für die Versicherung maßgebende Beschäftigung ausgeübt wurde. Die Ausnahmen (Ausstrahlung) betrafen u.a. Arbeitnehmer, die durch einen Betrieb, der in dem einen Staat seinen Sitz (Wohnsitz) hatte, zu einer vorübergehenden Beschäftigung in ein anderes Staatsgebiet entsandt wurden. Für die Dauer eines Jahres fanden die Rechtsvorschriften des Staates Anwendung, in dem der entsendende Betrieb seinen Sitz hatte (Wohnsitz des Arbeitgebers).

Die grundlegende Bestimmung wurde in Art. 4 verankert: „Die beiden Staaten stellen für die Leistungen aus den in Art. 1 bezeichneten Zweigen der Sozialversicherung den eigenen Staatsangehörigen und ihren Hinterbliebenen die Angehörige des anderen Staates und ihre Hinterbliebene gleich". Die einzige Ausnahme zu den Hauptleistungen stellten die Reichs-/Staatszuschüsse dar, über welche die Staaten frei bestimmen sollten (Art. 5, Art. 20).

Die zweite grundsätzliche Regelung fand ihre Widerspiegelung in den Artikeln 19, 23 und 26 über die Invalidenversicherung und Angestelltenversicherung. Die Vorschrift des Art. 20 Abs. 1 führte zunächst den Grundsatz der Zusammenrechnung der in den beiden Staaten zurückgelegten Beitragszeiten (soweit sie sich nicht deckten) für die

44 Szymanko, Polsko-niemiecka umowa o ubezpieczeniu społecznym (Der polnisch-deutsche Vertrag über die Sozialversicherung), Pr. i O. S. 1933, Nr. 2, S. 200 ff.; der paraphierte Text dieses Vertrags (Anfang 1928) war Vorbild für andere durch Deutschland abgeschlossene Verträge mit Jugoslawien, Österreich und der Tschechoslowakei; s. auch einen Kommentar von ders., Polsko-niemiecka umowa o ubezpieczeniu społecznym (Der polnisch-deutsche Vertrag über die Sozialversicherung), Pr. i O. S. 1933, Nr. 3, S. 259 ff.

45 Eichenhofer, Internationales Sozialrecht (Fußn. 31), S. 22 ff. 
Aufrechterhaltung und das Wiederaufleben von Anwartschaften und für die Erfüllung der Wartezeit ein. In ihrem Absatz 4 wurde der pro-rata-parta-temporis-Grundsatz eingeführt. Gemäß dieser Vorschrift gewährten die Versicherungsträger beider Staaten beim Eintritt eines Versicherungsfalles die Leistungen, auf die nach innerstaatlichen Vorschriften ein Anspruch bestand. Von dem Grundbetrag (und den Zuschüssen) war aber nur der Teil zu zahlen, der dem Verhältnis der innerstaatlichen Beitragszeit zur Summe der in den beiden Staaten zurückgelegten Beitragszeiten entsprach. Diese Kürzung trat nicht ein, wenn in einem der beiden Staaten nicht mehr als sechsundzwanzig Beitragswochen zurückgelegt wurden.

Die Bestimmungen über die Krankenversicherung haben im territorial und sachlich begrenzten Umfang den Grundsatz der Gegenseitigkeit realisiert. Entsprechend Art. 15 fanden im Verkehr zwischen den benachbarten Grenzgebieten der beiden Staaten die gesetzlichen Vorschriften über das Ruhen von Leistungen und über die Abfindung des Berechtigten keine Anwendung. Als benachbartes Grenzgebiet galt der Bezirk der Krankenkasse des anderen Staates, die mit dem Bezirk der für die Gewährung der Leistungen zuständigen Krankenkasse eine gemeinsame Grenze hatte. Erkrankte ein Versicherter in dem anderen Staat außerhalb des benachbarten Grenzgebietes, so hatte er Anspruch auf die ihm bei seiner Kasse zustehenden Leistungen, solange er seines Zustandes wegen nicht in das Gebiet des Staates zurückkehren konnte, in dem seine Krankenkasse ihren Sitz hatte (Art. 5 Abs. 5 und Art. 16).

Im Vertrag waren auch weitere wesentliche Bestimmungen enthalten, die auf Folgendes Bezug nahmen: verwaltungsmäßige Zusammenarbeit der Sozialversicherungsträger, die Einreichung von Rechtsmitteln, Betreuung seitens der Konsuln (Vertretungsbefugnisse) und die Einführung eines Schiedsverfahrens im Falle von Streitigkeiten betreffend die Handhabung des Vertrages. Sehr wichtig waren in der damaligen Lage die Schlussbestimmungen betreffend die Lösung von Problemen, die auf die Teilung der oberschlesischen Knappschaftsversicherung zurückzuführen waren.

\section{Die rechtliche Lage der polnischen Arbeiter im nationalsozialistischen} Staat 1933-1945

\section{Der Nationalsozialismus und das Arbeits- und}

Sozialversicherungsrecht 1933-1939

Während der Weltkrise kam die Einwanderung ausländischer Arbeiter nach Deutschland zum Stillstand. Ab 1936 stieg die Zahl der polnischen Arbeiter wieder an, was mit der Entwicklung der deutschen Wirtschaft, insbesondere wegen der Rüstungskonjunktur, verbunden war. Im Ergebnis der Verhandlungen, die auf Initiative der deutschen 
Regierung mit der polnischen Regierung aufgenommen worden waren, hat man folgende Zahlen der pro Jahr zugelassenen polnischen Landarbeiter festgelegt: für das Jahr $1937-10.000$, für $1938-60.000$, für $1939-90.000 .46$

In der Zeit nach 1933 bis zum 2. Weltkrieg ergingen keine direkt an polnische Landarbeiter gerichteten rechtlichen Regelungen. Insgesamt aber kam es im deutschen Arbeitsrecht zu gewaltigen Veränderungen, die die neue nationalsozialistische Ideologie verwirklichen und den Staat zu ihrer Umsetzung ausrüsten sollten. ${ }^{47}$ Eine skizzenhafte Darstellung einiger in dieser Zeit getroffenen Maßnahmen an dieser Stelle scheint deshalb wichtig zu sein, weil sie für ausländische Arbeiter insgesamt eine Bedeutung hatten (z. B. Regelungen aus dem Bereich der Beschäftigung und Gestaltung der Arbeitsbedingungen), aber darüber hinaus auch einen Ausgangspunkt für weitere Maßnahmen gegenüber einigen sog. rassisch fremden Nationalitäten, darunter auch Polen, in der Zeit des 2 . Weltkrieges bildeten.

Dazu zählen insbesondere die Ideologisierung des Arbeitsrechts mittels der Einführung der freien Gewerkschaften der Deutschen Arbeitsfront und des Gesetzes zur Ordnung der nationalen Arbeit vom 20. Juni 1934. Weiterhin sind die weit über die Bekämpfung der Arbeitslosigkeit hinausreichenden Maßnahmen im Bereich des Arbeitsmarktes zu berücksichtigen, die im Ergebnis mit einer völligen Kontrolle des Staatsapparats über den Arbeitsmarkt endeten (insbesondere das Gesetz vom 5. November 1935 über die Arbeitsvermittlung, Berufsberatung und Lehrstellenvermittlung, das Gesetz vom 26. Februar 1935 über die Einführung eines Arbeitsbuches, die Verordnung über die Beschränkung des Arbeitsplatzwechsels vom 1. September 1939 und die Verordnung zur Sicherstellung des Kräftebedarfs für Aufgaben von besonderer staatspolitischer Bedeutung [Dienstpflichtverordnung] vom 22. Juni 1938). Es ist auch auf die sehr rasch eingeführte staatliche Gestaltung und Kontrolle der Arbeitsbedingungen, insbesondere mittels der Institution der Reichstreuhänder der Arbeit, hinzuweisen, und schließlich auf die Pönalisierung des Arbeitsrechts, darunter auch der Arbeitsverhältnisse. Im letztgenannten Bereich ist die Einführung neuer, bisher im Arbeitsrecht nicht bekannter Haftungsmaßnahmen bemerkenswert, darunter auch solcher mit strafrechtlichem Charakter, sowie die Ausstattung der Reichstreuhänder der Arbeit mit repressiven Befugnissen, die bei jeder Zuwiderhandlung der sachlich sehr breit angelegten Anordnungen der Reichstreuhänder ausgeübt werden konnten. In schwereren Fällen der Pflichtverletzung konnte der Reichstreuhänder Strafverfahren vor dem Arbeitsgericht einleiten. Es sei in diesem Zusammenhang daran erinnert, dass die Verletzung der Pflichten aus dem Arbeitsverhältnis im bürgerlichen Recht im Allgemeinen nur privatrechtliche Folgen hatte, wie Fortfall des Lohnes, Schadenersatz oder fristlose Entlas-

46 Herbert, Geschichte der Ausländerbeschäftigung (Fußn. 1), S. 120.

47 Eine Darstellung des deutschen Arbeitsrechts in der Zeit der NS-Herrschaft bei Szurgacz, Przymusowe zatrudnienie Polaków przez hitlerowskiego okupanta w latach 1939-1945. Studium prawno-polityczne (Zwangsbeschäftigung der Polen während der Besatzung durch Hitler in den Jahren 1939-1945. Eine rechtspolitische Studie), 1971, S. 11 ff. 
sung. E. Jacobi hat 1922 in Bezug auf den Arbeitsvertragsbruch geschrieben: „Irgendeine Bestrafung oder Verwaltungsmaßregel tritt nicht ein“".48 Während des Krieges konnte die Polizei, einschließlich der Gestapo, einschreiten, weil die Arbeitsleistung als ein politisches Verhalten betrachtet wurde (Arbeit als Dienst an Volk und Staat).

Ebenso wie im Arbeitsrecht ist es im Bereich der Sozialversicherung in der Zeit bis zum Ausbruch des 2. Weltkrieges gegenüber den polnischen Arbeitern zu keinen Veränderungen gekommen. Insgesamt aber brachte die NS-Zeit den „,machtpolitischen Zugriff der NS-Partei auf die Institution der Sozialversicherung" mit sich. Es wurde die Selbstverwaltung aufgelöst, aus rassischen Gründen wurden Juden zunächst aus der Krankenversicherung und aus der kassenärztlichen Vereinigung, später aus der gesamten Sozialversicherung ausgeschlossen. ${ }^{49}$

II. Die Rechtslage der polnischen Arbeiter in der Zeit 1939-1945 im Reich und in den in das Reich eingegliederten Ostgebieten

\section{Die politischen Ziele der Naziherrschaft gegenüber Polen}

Der Krieg gegen Polen wurde mit dem Ziel einer dauerhaften Herrschaft über dieses Gebiet, aber auch über weitere östliche Gebiete begonnen. Am Anfang des Krieges war das Programm der Okkupationspolitik noch nicht vollständig ausgearbeitet, vieles hing von der weiteren politischen Entwicklung in Europa ab. Über einige Angelegenheiten herrschte jedoch Klarheit. Dazu zählte die Absicht, einen Teil des polnischen Territoriums in das Reich einzugliedern. Die Pläne in Bezug auf Restpolen schwankten von der Konzeption einer Art Reststaates, über eine Kolonie bis schließlich zum Deutschen Generalgouvernement Polen. ${ }^{50}$ Die Eingliederung der Ostgebiete wurde durch Erlass des Führers und Reichskanzlers über die Verwaltung der besetzten polnischen Gebiete vom 8. Oktober 1939 bestimmt. 51

Die eingegliederten Ostgebiete umfassten fast die Hälfte des durch das III. Reich besetzten polnischen Territoriums (ohne die durch die Sowjetunion besetzten Gebiete), d.h. ca. $90.000 \mathrm{~km}^{2}$ mit ca. 10 Millionen Einwohnern; sie wurden in die Reichsgaue Posen (später Reichsgau Wartheland) und Danzig-Westpreußen sowie Ostoberschlesien eingeteilt. 52

48 Jacobi, Einführung in das Gewerbe- und Arbeitsrecht. Ein Grundriß, 1928, S. 53 f.; s. auch Potthoff, Probleme des Arbeitsrechts (Fußn. 9), S. 118.

49 Peters, Die Geschichte der sozialen Versicherung, 1978, S. 105; Eichenhofer, Die Sozialversicherung (Fußn. 31), S. 34; Zacher, Aktuelle Herausforderungen (Fußn. 31), S. 92.

50 S. insbes. Madajczyk, Polityka III Rzeszy w okupowanej Polsce (Die Politik des III. Reiches im besetzten Polen), B. I, 1970.

51 RGBl. I, 1939, S. 2077.

52 Broszat, Nationalsozialistische Polenpolitik 1939-1945, 1961. 
Die Beschäftigung der Polen wie auch Angehöriger anderer Nationalitäten während des 2. Weltkriegs war für die Rüstungswirtschaft des III. Reiches eine wirtschaftliche Notwendigkeit. ${ }^{53}$ Die Stellung der ausländischen Zivilarbeiter in der Gesellschaft wie auch ihre Arbeits- und Lebensbedingungen waren durch die nationalistische Ideologie und durch Pläne, die die Naziherrschaft gegenüber den besetzten Gebieten und ihren Einwohnern ausgearbeitet hatte, bedingt. In dieser Hinsicht bildeten die Millionen im Reich eingesetzter Arbeiter ein vielfach gestaffeltes System. Auf der Stufenleiter hatten die sogenannten Gastarbeiter aus dem verbündeten Italien zusammen mit den Arbeitern aus Nord- und Westeuropa einen besseren Platz als die Polen. Eine noch niedrigere Stelle erhielten Ostarbeiter, Zigeuner und Juden. ${ }^{54}$

Die politischen Ziele gegenüber den Polen sind aus zahlreichen Äußerungen in den führenden Gremien des III. Reiches zu entnehmen. U.a. wurde bestimmt: „Die führende Bevölkerungsschicht in Polen soll so gut wie möglich unschädlich gemacht werden. Die restlich verbleibende niedrige Bevölkerung wird keine besonderen Schulen erhalten, sondern in irgendeiner Form herunter gedrückt werden. [...] Die primitiven Polen sind als Wanderarbeitnehmer in den Arbeitsprozess einzugliedern und werden aus den deutschen Gauen allmählich ausgesiedelt. [...] Ziel ist: Der Pole bleibt der ewige Saisonund Wanderarbeitnehmer [...] Der Pole ist als Arbeitskraft so lange auszubeuten, wie er gebraucht wird und dann sich selbst zu überlassen, und zwar so, dass er wirklich nur auf seine unzulänglichen Kräfte angewiesen ist“ ${ }^{* 55}$

\section{2. Über das Recht der Zwangsarbeit gegenüber Polen im Allgemeinen ${ }^{56}$}

In der Literatur aus der Kriegszeit wurde im Zusammenhang mit der Beschäftigung der Polen der Begriff „Sonderarbeitsrecht für Polen“ verwendet. 57 Ob dieser Begriff im Hinblick auf die Rechtslage der polnischen Arbeiter zutreffend war, wird an anderer Stelle erörtert. In diesem Aufsatz wird der Begriff „Recht der Zwangsarbeiter“ als Sammelbegriff für Regelungen verwendet, die Arbeitsbedingungen der Polen (Arbeits-

53 Herbert, Zwangsarbeit im „Dritten Reich” - ein Überblick, in: Barwig/Saathof/Wezde (Hrsg.), Entschädigung für NS-Zwangsarbeit. Rechtliche, historische und politische Aspekte, 1998, S. 17.

54 Herbert, Zwangsarbeit im „Dritten Reich” (Fußn. 53), S. 20.

55 Vollständiger Text bei Radziwończyk, „Akcja Tannenberg” grup operacyjnych Sipo i SD w Polsce jesienią 1939 (Die „Aktion Tannenberg” der Einsatzgruppen der Sipo und des SD in Polen im Herbst 1939), in: Przegląd Zachodni 1966, H. 5, S. 110; ders., Plany polityczne III Rzeszy wobec Polski i ich realizacja (Die politischen Pläne des III. Reiches gegenüber Polen und ihre Durchsetzung), in: Najnowsze Dzieje Polski 1968, B. XII, S. 6; s. auch Posener Tageblatt, 22.9.1939; Broszat, Zweihundert Jahre deutsche Polenpolitik, 1972, S. 278.

56 S. Szurgacz, Przymusowe zatrudnienie (Fußn. 47); s. auch eine diesbezügliche Dokumentation bei Konieczny/Szurgacz, Praca przymusowa Polaków pod panowaniem niemieckim 1939-1945 (Zwangsarbeit der Polen unter der deutschen Herrschaft 1939-1945), Documenta Occupationis B. X, 1976.

57 Küppers/Bannier, Arbeitsrecht der Polen im Deutschen Reich (Private Wirtschaft und öffentlicher Dienst), 1942; Küppers, Die Stellung des Polen im Arbeitsleben, RArbBl. V, 1941, S. 534. 
zeit, Entlohnung, Haftung usw.) festlegten und der auch für Regelungen der sogenannten polizeilichen Erfassung der polnischen Arbeiter galt. Die ersten Vorschriften aus dem letztgenannten Bereich hatten die Form von Anordnungen der Landräte und wurden auf die Verordnung vom 5. November 1939 über die Behandlung der Ausländer auf dem Gebiet des Deutschen Reiches gestützt. Im Hinblick auf die Perspektive einer Beschäftigung im Reich (in kurzer Zeit eine Million polnischer Arbeiter) wurde eine generelle Regelung ausgearbeitet. Am 8. März 1940 erließ der Ministerpräsident Generalfeldmarschall Göring, gleichzeitig Beauftragter für den Vierjahresplan und Vorsitzender des Ministerrats für die Reichsverteidigung, eine Anordnung über die Behandlung der Zivilarbeiter und -Arbeiterinnen polnischen Volkstums im Reich. Für die Ausführung der in der Anordnung enthaltenen Grundsätze wurden die im Einvernehmen tätigen $H$. Himmler als Reichsführer der SS und Chef der Deutschen Polizei und der Reichsarbeitsminister ermächtigt. Am selben Tage wurde eine Vielzahl von detaillierten Regelungen erlassen, die ein System der polizeilichen Erfassung der polnischen Arbeiter bildeten und die in der darauffolgenden Zeit nur wenig geändert wurden. ${ }^{58}$

Die Vorschriften über die polizeiliche Erfassung enthielten zweierlei Arten von Regelungen:

- solche, die eine gesellschaftliche Isolation der Polen zum Ziel hatten (Kennzeichnung jedes Kleidungsstückes mit dem Buchstaben »P«, Unterbringung getrennt von Deutschen, Separation der Polen vom Tisch, Verbot des Besuchs von Einrichtungen und Veranstaltungen kultureller, künstlerischer, kirchlicher, sportlicher und gesundheitlicher Art gemeinsam mit der deutschen Bevölkerung, Verbot des Besuchs von Badeanstalten, Gaststätten - außer ausdrücklich freigegebener - u.a.m.);

- solche, die die Bindung der Polen an den Aufenthaltsort und eine ständige Überwachung zum Ziel hatten (Aufenthaltszwang am Arbeitsort, der nur ausnahmsweise mit besonderer polizeilicher Genehmigung aufzuheben war, Verbot der Benutzung öffentlicher Verkehrsmittel, Verbot der Unterhaltung von Fahrrädern, Polizeistunde, Verbot der Eheschließung, Meldepflicht der Arbeitgeber über durch Polen begangene Zuwiderhandlungen u.a.m.).

Für eine gemeinsame Betrachtung der Vorschriften der polizeilichen Erfassung, mit denen des sogenannten Sonderarbeitsrechts im Rahmen eines allgemeinen Rechts der Zwangsarbeit gibt es folgende Argumente:

- Beide Regelungsgruppen sind funktionell eng miteinander verbunden; in gewisser Weise war die polizeiliche Erfassung eine Vorbedingung für die Erfüllung „arbeitsrechtlicher“ Pflichten (i.e.S.) der Polen. Übrigens ging die Gestaltung der polizeilichen Erfassung der „arbeitsrechtlichen“ im engeren Sinne zeitlich voraus.

58 Für eine diesbezügliche Dokumentation s. Konieczny/Szurgacz, Praca przymusowa (Fußn. 56), S. 3 ff. 
- Die Vorschriften aus dem Bereich der polizeilichen Erfassung beinhalteten arbeitsrechtliche Bestimmungen, indem sie die Fälle der Arbeitsvertragsbrüche der Polen grundsätzlich einem polizeilichen Strafverfahren unterwarfen.

- Einige „arbeitsrechtliche“ Regelungen wurden mit der vorherigen Zustimmung des Reichsführers der SS erlassen (z.B. die über Heimaturlaub).

Das Recht der Zwangsarbeit war Bestandteil eines allgemeineren rechtlichen Sondersystems, das unter der Bezeichnung „Polenstatut“ nicht nur für die Kriegsdauer, sondern auch für die weitere Zukunft gestaltet wurde. 59 Das Recht der Zwangsarbeit war im Rahmen dieses Polenstatuts der am meisten entwickelte Teil. Dies stimmte mit der schon erwähnten These der Nazis überein, dass die Polen nur als Arbeitskräfte zu betrachten seien.

Das Recht der Zwangsarbeit ist nicht im Wege einer einmaligen legislatorischen Entscheidung zustande gekommen. In Bezug auf das sogenannte Sonderarbeitsrecht der Polen war das vielmehr ein Entwicklungsprozess, der bis 1942 andauerte. Im Reich hat er mit der Reichstarifordnung für landwirtschaftliche Arbeitskräfte nichtdeutscher Volkszugehörigkeit vom 8. Januar 1940 begonnen. In den eingegliederten Gebieten wurden die Arbeitsbedingungen der polnischen Beschäftigten in Verordnungen der Reichsstatthalter (Oberpräsidenten), später in Anordnungen der Reichstreuhänder der Arbeit geregelt. In den dort festgelegten Arbeitsbedingungen gab es Unterschiede in den einzelnen Gauen und zwischen den eingegliederten Ostgebieten und dem Reich. Diese Unterschiede wurden jedoch nivelliert und schon am 5. Oktober 1941 wurde eine einheitliche Regelung für das Gebiet des Reiches und der eingegliederten Gebiete in der Form einer Anordnung des Reichsarbeitsministers über die arbeitsrechtliche Behandlung der polnischen Beschäftigten geschaffen. Für die in öffentlichen Verwaltungen und Betrieben beschäftigten Polen wurde eine ähnliche Regelung Anfang 1942 erlassen. Die in diesen Anordnungen getroffenen grundsätzlichen Regelungen wurden später nur noch in Details geändert.

\section{Einige Institutionen des Rechts der Zwangsarbeit}

\section{a) Entstehung und Auflösung des Beschäftigungsverhältnisses}

Wie schon erwähnt, wurde in Deutschland mit Hilfe der Gesetze über die Arbeitsvermittlung (1935) und über die Einführung eines Arbeitsbuches (1935) schon vor dem Kriege eine völlige Kontrolle des Staates über den Arbeitsmarkt erreicht. Die Verord-

59 Von Rosen-von Hoewel, Das Polenstatut, Deutsche Verwaltung 1942, H. 6, S. 109: „Der Nationalsozialismus hat in Anknüpfung an die frühgermanische Zeit die blutmäßig bestimmten Unterschiede zwischen den Völkern und den einzelnen Menschen klar herausgearbeitet. Er geht deshalb nicht, wie der Liberalismus, von der Gleichheit aller Menschen aus, sondern gerade von ihrer Ungleichheit, die in ihren naturgegebenen rassischen, geistigen und körperlichen Anlagen begründet ist. Dementsprechend müssen sie je nach ihrem Wert für den Fortbestand des Volkes auch verschiedene Rechte und Pflichten haben". 
nung über die Beschränkung des Arbeitsplatzwechsels vom 1. September 1939 hatte eine Kündigung des Arbeitsverhältnisses wie auch eine Neueinstellung von der vorherigen Zustimmung des Arbeitsamts abhängig gemacht. Was die sich im Reich befindlichen polnischen Landarbeiter anbelangt, so hat sich der Reichsarbeitsminister schon Anfang Dezember „damit einverstanden erklärt, dass die Rückpflichtigen polnischen Arbeitskräfte bis auf weiteres im Inlande verbl[ie]ben“.

Im Bereich der Beschäftigung der polnischen Arbeiter wurden zu Anfang des Krieges in begrenztem Umfang Formen der freiwilligen Rekrutierung zur Arbeitsaufnahme im Reich angewendet. In den eingegliederten Ostgebieten wurde zusätzlich die Dienstverpflichtung eingeschaltet. Die rechtlichen Mittel der Dienstverpflichtung wurden jedoch nur begrenzt eingesetzt, grundsätzlich wurden Methoden einer Zwangsrekrutierung zum Arbeitseinsatz im Reich angewendet. 60

In Bezug auf die rechtliche Lage der polnischen Arbeiter im Bereich ihrer Beschäftigung ist festzustellen, dass in dem Zeitpunkt, als die Polen gemäß den Vorschriften über die Arbeitsvermittlung durch das Arbeitsamt in einer Kartei erfasst wurden, sie gleichzeitig die Verfügungsmacht über ihre eigene Person verloren. Über ihre Einstellung hat das Arbeitsamt nach eigenen Kriterien entschieden. Die Verfügungsmacht endete nicht mit dem Zeitpunkt einer Beschäftigung, sondern dauerte ununterbrochen während des ganzen Aufenthalts im Reich.

In diesem Zusammenhang scheint die Schlussfolgerung berechtigt zu sein, dass die polnischen Arbeiter an den ihnen zugeteilten Arbeitsplatz rechtlich gebunden waren. Der Generalgouverneur H. Frank schrieb Ende 1943 an F. Sauckel: „Die Erfassung der Polen für den Arbeitseinsatz im Reich erfolgt bedingungslos, ihre Einweisung in Arbeit ohne jede persönliche Mitwirkung. Ihre Verpflichtung zur Arbeitsleistung im Reich ist zeitlich unbegrenzt. Auch für den Fall schwerwiegender Gründe familiärer Art besteht für sie keine Gewähr für eine Rückkehr in die Heimat. Der Einsatz im Reich hat somit für die Polen in wesentlicher Hinsicht den Charakter einer der Gefangenschaft ähnlichen Freiheitsbeschränkung"61

Im Bereich der Beschäftigung äußerte sich seit jeher die Schutzfunktion des Arbeitsrechts, indem Beschäftigungsverbote hinsichtlich des Alters bzw. des Geschlechts der Arbeitnehmer eingeführt wurden. In Deutschland z. B. war es das Gesetz über die Kinderarbeit und die Arbeitszeit der Jugendlichen (Jugendschutzgesetz vom 30. April 1938) und das Mutterschutzgesetz von 1927.

Die Anwendung des Jugendschutzgesetzes gegenüber jugendlichen Polen in den eingegliederten Ostgebieten wurde ausdrücklich ausgeschlossen. Dies bedeutete die Gleichstellung der jugendlichen Polen mit Erwachsenen. Im Reich galt das Jugend-

60 In der deutschen Literatur s. Schminck/Gustavus, Zwangsarbeit und Faschismus. Zur „Polenpolitik” im „Dritten Reich”, Kritische Justiz 1980, S. 1 ff., 184 ff.

61 S. Mitteilung des Generalgouverneurs Frank an den Generalbevollmächtigten für den Arbeitseinsatz Sauckel betr. der Behandlung der im Reich eingesetzten Arbeitskräfte polnischen Volkstums, abgedruckt in: Documenta Occupationis (Fußn. 56), S. 306. 
schutzgesetz anfangs auch gegenüber Polen. In der Anordnung vom 5. Oktober 1941 wurden polnische Beschäftigte im Alter von 14 bis 18 Jahren grundsätzlich mit den erwachsenen Polen gleichgestellt (§ 14). Das Jugendschutzgesetz sollte danach nur noch für polnische Kinder gelten. Am 12. Juli 1942, also in der Zeit, als im Reich immer mehr polnische Kinder beschäftigt wurden, gab der Generalbevollmächtigte für den Arbeitseinsatz F. Sauckel einen Runderlass unter dem Titel „Beschäftigung polnischer Kinder und Kinder aus den besetzten Ostgebieten in gewerblichen Betrieben" heraus, in dem u.a. folgende Auslegung des Anwendungsbereiches des Jugendschutzgesetzes auf polnische Kinder enthalten war: „Es besteht jedoch keine Veranlassung, den Kinderschutz für polnische Kinder ebenso wie für deutsche Kinder durchzuführen, da die Vorschriften des Jugendschutzgesetzes die deutschen Kinder ja nicht nur gegen Überanstrengungen bei der Arbeit schützen, sondern ihnen auch den erfolgreichen Besuch der Schule sichern sollen. Von der Ausstellung von Kinderkarten für polnische Kinder kann in der Regel abgesehen werden. Es bestehen auch keine Bedenken, wenn polnische Kinder über die im Jugendschutzgesetz vorgesehenen Grenzen hinaus beschäftigt werden." 62

Was die polnischen Frauen betraf, so war das Mutterschutzgesetz aus dem Jahre 1927 auf sie aufzuwenden, was der Reichsarbeitsminister in seinem Runderlass vom 7. Juli 1941 bestätigte. Gleichzeitig jedoch wurde in diesem Erlass bestimmt, dass das Ausmaß des gesetzlich gewährten Schutzes gegenüber den polnischen Frauen geringer sein durfte als der den deutschen Frauen gewährte Schutz. Die genauen Grenzen des den polnischen Frauen zustehenden Schutzes wurden nicht präzisiert. Das neue Mutterschutzgesetz aus dem Jahre 1942, das den deutschen Frauen trotz des Krieges einen erhöhten Arbeitsschutz gewährt hatte, sollte auf die polnischen Arbeiterinnen nur in den Grenzen eines Mindestschutzes angewendet werden. Dieser Mindestschutz wurde im Reichsgau Wartheland als noch zu hoch eingeschätzt. Ein vertrauliches Schreiben des GBA aus dem Jahre 1943 schränkte diesen Mindestschutz ein. ${ }^{63}$

\section{b) Erholungsurlaub}

In der Zwischenkriegszeit wurde in allen europäischen Ländern ein Erholungsurlaub für Arbeiter in der Form einer in Gesetzen bzw. Tarifverträgen gewährten bezahlten Freizeit für Erholungszwecke eingeführt. Während des Krieges wurden in Deutschland die Urlaubsvorschriften nur für eine kurze Zeit suspendiert (4.9.1939-17.12.1939); sonst wurde bis 1943 das Niveau aus der Vorkriegszeit erhalten. Auch 1943 wurden keine drastischen Begrenzungen eingeführt.

Die für polnische Landarbeiter geltende Reichstarifordnung enthielt keine Urlaubsvorschriften, was in der damaligen rechtlichen Situation bedeutete, dass diesen Arbeitern kein Recht auf Urlaub zustand. Den sonstigen polnischen Arbeitern wurde das

62 Dazu näher Szurgacz, Przymusowe zatrudnienie (Fußn. 47), S. 84.

63 Szurgacz, Przymusowe zatrudnienie (Fußn. 47). 
Recht auf Urlaub nicht untersagt. Im März 1941, also in dem Zeitpunkt, als gemäß den geltenden Urlaubsvorschriften die Polen einen Anspruch auf Urlaub erlangen konnten, wurde das Ruhen der Urlaubsansprüche für polnische Beschäftigte eingeführt. Gemäß der Anordnung des Reichsarbeitsministers über Urlaub der im Reich eingesetzten Zivilarbeiter/-innen polnischen Volkstums wurde den Polen das Recht auf Urlaub nicht untersagt. Das eingeführte Ruhen der Urlaubsansprüche, das mit den Transportschwierigkeiten begründet wurde, sollte nur für eine Übergangszeit gelten. Bis Kriegsende wurde es jedoch nicht aufgehoben.

\section{c) Den polnischen Arbeitern gegenüber angewandtes Maßnahmensystem}

Gegenüber polnischen Arbeitern konnten anfangs dieselben Maßnahmen, wie sie für deutsche Arbeitnehmer vorgesehen waren, angewendet werden. Für die polnischen Zwangsarbeiter wurde jedoch in kurzer Zeit im Wege nicht veröffentlichter Rechtsakte ein gesondertes System eingeführt, das das „klassische“ Haftungssystem zwar nicht ausschloss, jedoch in der Praxis die Hauptrolle spielte. Die wesentlichsten Merkmale dieses Systems sind die folgenden:

(1) Seit der Anordnung H. Himmlers vom 23. Dezember 1939 über die Behandlung arbeitsunwilliger polnischer Arbeiter, deren Gegenstand die Behandlung der Arbeitsniederlegung war, wurde die Zuständigkeit der Polizei bei den durch die Polen verursachten Pflichtverletzungen ständig erweitert. So wurde die Polizei schon Anfang 1940 für Fälle der Arbeitsniederlegung, der ständig lässigen Arbeit, der Aufhetzung der Arbeiter und der unerlaubten Kontakte mit Deutschen zuständig.

(2) Allein zuständig war die Geheime Staatspolizei.

(3) Trotz einiger Behauptungen im gegenwärtigen Schrifttum, dass die Polizei diese Kompetenzen sich selbst aneignete, ist festzustellen, dass eine Vereinbarung zwischen dem Chef der Deutschen Polizei, H. Himmler, und dem Reichsarbeitsminister getroffen wurde, wonach Fälle des Arbeitsvertragsbruchs polnischer Arbeiter der Gestapo überlassen wurden. Der Reichsarbeitsminister erteilte den Reichstreuhändern der Arbeit und den Leitern der Arbeitsämter entsprechende Weisungen.

(4) Es waren folgende Maßnahmen vorgesehen:

- polizeiliche Warnung,

- kurzfristige Polizeihaft,

- Einweisung in ein Arbeitserziehungslager der Gestapo,

- Einweisung in ein Konzentrationslager,

- Sonderbehandlung (die die Todesstrafe durch Erhängen bedeutete).

(5) Im Rahmen dieses Systems wurden Arbeitnehmerpflichtverletzungen mit VerstöBen gegen andere den Polen auferlegte Pflichten und Verbote aus dem Bereich der sogenannten polizeilichen Erfassung zusammengefasst und im einheitlichen polizeilichen Verfahren verfolgt.

(6) Die Bedeutung der Amtsgerichte ging infolge der Übernahme der Angelegenheiten durch die Polizei zurück. Demgegenüber wurden die Sondergerichte ein wesentli- 
ches Element des Systems, in dem drakonische Strafen verhängt wurden. Der Generalstaatsanwalt in Breslau schrieb am 10. März 1943 etwa: „Bisher ist das Sondergericht in Breslau davon ausgegangen, dass die sich an einem deutschen Betriebsführer oder Arbeitsführer tätlich vergehen, die Todesstrafe verwirkt haben" 64

(7) Die Praxis kannte noch zahlreiche andere Methoden der Bestrafung der polnischen Zwangsarbeiter, von denen in vertraulichen Anweisungen besonders das Verprügeln empfohlen wurde.

M.E. ist das hier nur in seinen Grundzügen skizzierte System mit der im Arbeitsrecht bekannten Haftung des Arbeitnehmers, die mit zahlreichen, den Arbeitnehmer schützenden Bestimmungen ausgestattet war, nicht zu vergleichen. Im Grunde genommen handelte es sich um ein System des physischen und psychischen Terrors und einer rücksichtslosen Beseitigung derjenigen polnischen Arbeiter, die versuchten, einen Widerstand zu leisten.

d) Die Sicht der rechtlichen Lage der polnischen Zwangsarbeiter in der rechtswissenschaftlichen Literatur

Die Rechtslage der polnischen Zwangsarbeiter wurde während des Krieges in zahlreichen Beiträgen kommentiert. Dies war notwendig wegen der sehr komplizierten rechtlichen Regelungen, wie auch zur Begründung der eingeführten diskriminierenden Maßnahmen. Die in diesen Beiträgen unternommene Rationalisierung der eingeführten Lösungen diente zugleich als Auslegungsgrundlage bei allen anderen artverwandten Fragen.

Die Begründung des rechtlichen Sonderstatus basierte auf rassischen Argumenten und knüpfte an die Ziele der deutschen Nationalpolitik im Osten an. ${ }^{65}$

Dieselben Argumente wurden bei der Begründung des Ausschlusses der Polen aus dem Geltungsbereich des Gesetzes zur Ordnung der Nationalen Arbeit (AOG) verwendet. W. Herschel schrieb 1942: „Das deutsche Arbeitsrecht gilt bis zu einem gewissen Grade auch für Ausländer, die bei uns beschäftigt sind. Mag sich auch in manchen Punkten ein Ausländer in die Gemeinschaft des Betriebes und des Unternehmens eingegliedert haben, der somit auch Genosse eines Arbeitsverhältnisses sein kann. Das wird auch von der Rechtsordnung anerkannt. Nun gibt es aber Menschen, die insbesondere als Träger eines völlig artfremden Blutes oder einer artfremden Haltung, unserem völkischen Rechtsempfinden so fern stehen, dass zwischen ihnen und Deutschen eine auf Ehre, Kameradschaft und Treue gegründete Gemeinschaft und daher ein Arbeitsverhältnis ausgeschlossen ist" ${ }^{\prime 66}$.

64 Reichsjustizministerium, Mikrofilm 24769, abgedruckt in: Documenta Occupationis (Fußn. 56), S. 212.

65 Von Rosen-von Hoewel, Das Polenstatut (Fußn. 59), S. 109 ff.

66 Herschel, Beschäftigungsverhältnisse eigener Art, Deutsches Recht 1942, S. 1455. 
Die Nichtanwendung der grundlegenden arbeitsrechtlichen Gesetze des Nationalsozialismus auf polnische Beschäftigte hatte weitgehende Konsequenzen. Wie bereits erwähnt, hatte das AOG die sogenannte Betriebsgemeinschaft zwischen dem Unternehmer (Betriebsführer) und den Angestellten und Arbeitern als Gefolgschaft begründet, die zur zentralen Wertgrundlage und zum Gestaltungsmodell des ganzen Arbeitsrechts erhoben wurde. Jedes einzelne Arbeitsverhältnis wurde zu einem Gliedschaftsverhältnis innerhalb der Volksgemeinschaft.

Im Endeffekt wurden die polnischen Beschäftigten außerhalb jeglicher Gemeinschaft und außerhalb der deutschen Sozialverfassung gestellt. Sie hatten kein Recht auf die Fürsorge, die ein wesentlicher Inhalt des Arbeitsrechts war. Nach W. Herschel standen die polnischen Beschäftigten in einem „Beschäftigungsverhältnis eigener Art“, das sich um einen schuldrechtlichen „Austauschvertrag“ drehte, der sich an das Dienstvertragsrecht des BGB (§§ 611 ff.) anlehnte, jedoch diesem Recht nicht ohne Weiteres und ausnahmslos unterstand. ${ }^{67}$ Auch $A$. Hueck sah in dem Beschäftigungsverhältnis kein Arbeitsverhältnis im Sinne des nationalsozialistischen Arbeitsrechts, sondern einen schuldrechtlichen Austausch von Arbeitsleistung und Lohn. 68

Den bereits zitierten theoretischen Auffassungen ist insoweit Rechnung zu tragen, als dass sie das Bestehen eines Arbeitsverhältnisses in der nationalsozialistischen Prägung verneinten. Es bestand auch kein Arbeitsverhältnis im klassischen Sinne. Selbst ein schuldrechtliches Austauschverhältnis ist auszuschließen. M.E. sollte diese rechtliche Lage als Zwangsbeschäftigungsverhältnis, das durch ein spezielles Recht der Zwangsarbeit geregelt wurde, bezeichnet werden.

Die die Beschäftigung der Polen betreffenden Regelungen waren ein wichtiges Instrument zur Durchführung der Zwangsarbeit. Im Rahmen dieses Aufsatzes ist es nicht möglich, zusätzlich die Praxis zu schildern. In einigen Fällen wich sie von diesen Regelungen $a b$. Es gab Fälle milderer Handhabung dieser Vorschriften, sogar der Nichtanwendung als Ausdruck der Ablehnung der offiziellen Einstellung. Andererseits aber gab es auch Fälle der Behandlung der Polen, die in eine ganz andere Richtung zielten und als extrem zu bezeichnen sind. In den Erinnerungen der polnischen Zwangsarbeiter wurden beide der soeben skizzierten Situationen dokumentiert. Aus der Sicht der damals geltenden Regelungen waren sie ein Verstoß gegen die Vorschriften, die einen zwingenden Charakter hatten. Im ersten Falle konnte die zuständige Behörde einschreiten (das Arbeitsamt bzw. die Polizei) und den Polen an einen anderen Arbeitsplatz versetzen bzw. gegen den deutschen Arbeitgeber einen Strafantrag stellen. Im zweiten Falle konnte es mit der Versetzung des polnischen Arbeiters an einen anderen Arbeitsplatz enden.

67 Herschel, Beschäftigungsverhältnisse (Fußn. 66), S. 1455.

68 Hueck, Deutsches Arbeitsrecht. Ein Grundriss, 1944. 


\section{Die Lage der polnischen Arbeiter in der Sozialversicherung}

Was die Lage der polnischen Arbeiter in der Sozialversicherung in der Zeit des 2. Weltkrieges betrifft, so ist zwischen dem Territorium des III. Reiches und den in das Reich eingegliederten Ostgebieten zu unterscheiden.

Auf dem Gebiet des Reiches galten für polnische Arbeiter die uns schon bekannten Regelungen aus der Zeit vor 1939 weiter. Im Gegenteil zum Arbeitsrecht wurde auf dem Gebiet des Reichs kein umfassendes „Sondersozialversicherungsrecht für Polen“ eingeführt. Der polnische Forscher W. Rusinski vertritt die Meinung, wonach die Gründe dafür, dass keine besondere Sozialversicherung für die Polen im Reich eingeführt wurde, auf technische Schwierigkeiten für so ein Vorhaben zurückzuführen seien. ${ }^{69}$ Diese Stellungnahme bedarf jedoch zweier Ergänzungen. Zum einen sollte darauf hingewiesen werden, dass für die Arbeit im Reich nur gesunde Polen eingesetzt und die Kranken und Hilfsbedürftigen in das Generalgouvernement rückgeführt werden sollten. ${ }^{70}$ Zum anderen war es beabsichtigt, in der Sozialversicherung ein Sondersystem zunächst in den eingegliederten Ostgebieten einzuführen, das erst später auf das Reichsgebiet erweitert werden sollte. ${ }^{71}$

In den eingegliederten Ostgebieten wurden sehr schnell grundsätzliche Änderungen eingeführt. Einerseits wurde die polnische Sozialversicherung stufenweise durch die deutsche ersetzt. Die Einführung der Reichsversicherung in Oberschlesien erfolgte am 16. Januar 1940 und in den restlichen Gebieten am 22. Dezember 1941 mit der Verordnung über die Einführung der Reichsversicherungsordnung in den eingegliederten Ostgebieten. ${ }^{72}$ Die Sozialversicherungsvorschriften enthielten von Anfang an Polen diskriminierende Regelungen. Polnische Renten wurden Personen untersagt, die eine deutschfeindliche Tätigkeit ausübten, im Warthegau wurde die Auszahlung der Renten eingestellt, neue Rentenanträge wurden nicht bearbeitet, das Krankengeld wurde erst ab einer viertätigen Erkrankung gezahlt. ${ }^{73}$

Im Jahre 1941 wurden in vertraulichen parteilichen Mitteilungen die Merkmale des zukünftigen Systems für Polen umrissen. Es wurde mitgeteilt, dass ,[d]ie demnächst zu erwartende Regelung des Reichsarbeitsministers [...] auf folgenden Grundsätzen [beru-

69 Rusiński, Położenie robotników polskich w latach wojny 1939-1945 na terenie Rzeszy i „obszarów wcielonych", B. II, 1955.

70 Rusiński, Położenie robotników (Fußn. 69), S. 235 und der dort zitierte Erlass des Generalbevollmächtigten für den Arbeitseinsatz vom 27. August 1942 über die Rückführung Geisteskranker und anderer Hilfsbedürftiger in das Generalgouvernement sowie der Erlass vom 25. September 1942 über die Rückführung erkrankter und arbeitsunfähiger polnischer Arbeiter in das Generalgouvernement.

71 Darüber informierte der Generalgouverneur H. Frank in der Mitteilung vom 21. September 1943 (Fußn. 55).

72 RGBl. I, 1941, S. 777.

73 Szurgacz, Das Polenstatut in der Sozialversicherung in den in das III. Reich eingegliederten Ostgebieten (1939-1945), in: Ruland/v. Maydell/Papier (Hrsg.), Verfassung, Theorie und Praxis des Sozialstaats, Festschrift für Hans F. Zacher zum 70. Geburtstag, 1998, S. 885 ff. 
he]: Alle Leistungen werden nur als Unterstützungen und ohne Rechtsanspruch gewährt. Gleichwohl müssen die Schutzangehörigen und Staatenlosen polnischen Volkstums die vollen reichsgesetzlichen Beiträge entrichten. Die Unterstützungen sind nur bis zur Höhe der für Unterstützungsempfänger maßgebenden Richtsätze der öffentlichen Fürsorge auszuzahlen. Alle Leistungen, die als bevölkerungspolitische Maßnahmen anzusehen sind, werden aufs äußerste eingeschränkt [...] Eine Reihe von Leistungen der reichsgesetzlichen Unfallversicherung, insbesondere Leistungen mit bevölkerungspolitischer Zielsetzung, kommen für Schutzangehörige und Staatenlose polnischen Volkstums überhaupt nicht in Betracht"74

Die seit längerer Zeit angekündigte Regelung der besonderen Lage der Polen in der Sozialversicherung erfolgte durch Erlass des Reichsarbeitsministers vom 26. August 1942 über die den Schutzangehörigen und Staatenlosen polnischen Volkstums in den eingegliederten Ostgebieten an Stelle der Leistungen der Reichsversicherung zu gewährenden Unterstützungen.

Im Erlass wurden zunächst Vorschriften der Verordnung vom 22. Dezember 1941 aufgezählt, die keine Anwendung auf Polen fanden. An zweiter Stelle wurde der Charakter der an Polen zu gewährenden Leistungen bestimmt und an dritter Stelle der Umfang der ihnen zustehenden Leistungen geregelt.

Nicht auf Polen anzuwenden waren die Vorschriften der Verordnung vom 22. Dezember 1941, welche für Deutsche in den eingegliederten Gebieten gewisse Privilegien eingeführt hatten. Entsprechend dem $\S 2$ des Erlasses sollten die Leistungen an Polen vom Stichtag an (d.h. dem 1. Oktober 1942) als Unterstützungen gewährt werden; auf diese bestand kein Rechtsanspruch. Für die Zeit vor dem Stichtag sollten weder Leistungen noch Unterstützungen gewährt werden; soweit Leistungen für die Zeit vor dem Stichtag gezahlt worden waren, sollten diese weiterhin bestehen bleiben.

In den Abschnitten II bis IV des Erlasses wurden Grundsätze der Erteilung von Unterstützungen im Falle der Krankheit, des Unfalles (Berufskrankheit), der Invalidität und Tod unter Zurücklassung von Hinterbliebenen geregelt. Unterstützungen durften nur bis zur Höhe des für den Schutzangehörigen oder Staatenlosen polnischen Volkstums an seinem Wohnort beim Beginn der Unterstützungszahlung geltenden Richtsatzes der öffentlichen Fürsorge gewährt werden. Dieser Richtsatz blieb für die ganze Dauer der Unterstützungszahlung maßgebend ( $§ 2$ Abs. 2). Am 13. Oktober 1942 erklärte der Reichsarbeitsminister ergänzend, dass es sich dabei um den Richtsatz handle, der für den Polen selbst gelte. Nicht zu berücksichtigen waren Richtsätze für Familienangehörige des Empfängers oder Familienzuschläge.

Die Art und das Ausmaß der den Polen zustehenden Leistungen kann im Rahmen dieses Aufsatzes nur skizzenhaft dargestellt werden. Auch auf die Unterschiede in der Behandlung im Verhältnis zu deutschen Versicherten kann hier nicht näher eingegangen werden.

74 S. Vertrauliche Informationen 1942, Nr. 13, B. 253, abgedruckt bei: Rusiński (Fußn. 69), S. 196 Fußn. 60. 
Im Falle der Krankheit sollten gemäß $\S 3$ des Erlasses als Unterstützung nur die Regelleistungen gewährt werden. Orthopädische Versorgung, Zahnprothesen und Zahnbrücken wurden nicht geleistet. Die Krankenhauseinweisungen erfolgten als Kennleistungen. Das Krankengeld wurde nur zur Hälfte und erst vom achten Tag der Arbeitsunfähigkeit an gezahlt, d.h., dass ein Pole von der Krankenkasse 25\% des Grundlohnes bis höchstens 300 RM (monatlich höchstens 75 RM) bei einer Wartezeit von acht Tagen ohne Entgelt erhalten konnte. Trotz dieser niedrigen Leistungen zahlten Polen 5\% vom Bruttoeinkommen an die Krankenversicherung (gegenüber 3,5 bis 4,5\% bei den deutschen Versicherten). Die Familienhilfe wurde auf die unbaren Leistungen beschränkt, d.h. den Familienangehörigen wurden überhaupt keine finanziellen Leistungen wie Krankenhauskosten, Operationskosten u.Ä. gewährt, sondern nur die ärztliche Behandlung bezahlt. Bei der Wochenhilfe entfielen ebenso Barleistungen. Kindern sollten Unterstützungen nur bis zum vollendeten 15 . Lebensjahr (18. bei Deutschen) gezahlt werden. Sterbegelder wurden nicht gewährt.

Bei Unfällen (Berufskrankheiten) wurde den Verletzten eine Unterstützung zugesprochen, die jedoch in wesentlich engeren Grenzen als bei den Deutschen erfolgen konnte. Die Zahlung einer Unterstützung wurde nämlich überhaupt nur unter der Voraussetzung einer Erwerbsbeschädigung von 33,33\% vorgesehen; bei Deutschen waren es $20 \%$. Die Unterstützung in Höhe des vollen Richtsatzes der Fürsorge wurde erst dann gewährt, wenn die Erwerbsfähigkeit des Verletzten um die Hälfte gemindert war, 2/3 bei Minderung der Erwerbsfähigkeit um mindestens 1/3; bei einer Erwerbsminderung von weniger als $1 / 3$ sollte keine Unterstützung gewährt werden ( $\$ 4$ Abs. 1).

Die Höhe der Richtsätze der Fürsorge wurde bereits genannt. Als Berechnungsbasis für die deutsche Rente diente dabei das gesamte Jahreseinkommen vom Unfalltag an zurückgerechnet.

Bezüglich der Hinterbliebenenfürsorge wurde in einer Denkschrift des Oberschlesischen Instituts für Wirtschaftsforschung festgestellt; „Die größte Härte trifft die Hinterbliebenen von tödlich Verletzten“. Gemäß $\S 4$ Abs. 1 Pkt. 2 des Erlasses erhielt die Witwe des Getöteten als Unterstützung die Hälfte des Richtsatzes der Fürsorge, solange sie invalide war oder mindestens vier Kinder unter fünfzehn Jahren erzog. Um das Ausmaß der Diskriminierung der Polen in diesem Bereich zu zeigen, wird in der Denkschrift des Oberschlesischen Instituts folgendes Beispiel zitiert: „Eine deutsche Witwe mit drei Kindern bekommt $80 \%$ des Jahreseinkommens ihres verunglückten Mannes ausgezahlt, d.h. bei RM 2000 Lohn eine Rente von 1600. Die Polin würde nichts erhalten" 75 .

Entsprechend $\S 4$ Abs. 2 waren Kinderzulage, Sterbegeld, Witwenabfindung, Witwenrente, Waisenrente, Aszendentenrente, Witwenbeihilfe, Pflege, Berufsfürsorge, Tages- und Familiengeld sowie Kannleistungen nicht zu gewähren.

75 Oberschlesisches Institut für Wirtschaftsforschung (Hrsg.), Die Bedeutung des Polen-Problems für die Rüstungswirtschaft Oberschlesiens, 1944, abgedruckt in: Documenta Occupationis Teutonice, I, 1945, S. 135 f. 
Die Härten dieser Regelung erweckten in den industriellen Kreisen Oberschlesiens Kritik. Im Zusammenhang mit den Regelungen über die Behandlung im Falle der Krankheit wurde darauf hingewiesen, dass die Regelung, nach der das Krankengeld den Polen erst vom achten Tage der Arbeitsunfähigkeit an gezahlt wurde, häufig dazu führte, dass Familienväter sich erst dann krank meldeten, wenn der Fall bereits schon sehr ernst geworden war. Die Regelung, die mit der Absicht getroffen wurde, die Polen vor unberechtigtem Krankfeiern zurückzuschrecken, bewirkte infolgedessen nicht selten das genaue Gegenteil, weil der betreffende Mann durch die Verschlimmerung seiner Krankheit für den Betrieb viel länger ausfiel, und somit den gesamten Produktionsprozess belastete.

Bezüglich der Hinterbliebenenfürsorge wurde von den Werken, insbesondere den Grubenverwaltungen, darauf hingewiesen, dass die niedrigen Unfallsätze und noch mehr der Mangel einer wirksamen Hinterbliebenenfürsorge „,die polnischen Arbeitskräfte verständlicherweise davor zurückschrecken lassen, sich bei gefährlichen Arbeiten voll einzusetzen. Sie wissen, dass ihre Familie bei ihrem Unfall mehr oder minder dem Hunger ausgesetzt ist und suchen sich und ihre Familie durch äußerste Vorsicht vor dieser Gefahr zu schützen“776.

Diese Bedenken wurden rein ökonomisch motiviert. In einigen Werken wurde jedoch aus humanitären Gründen versucht „im Rahmen des Möglichen zu helfen“, und zwar durch Lieferung von Invalidenkohle oder durch Gewährung von Freikohle an Witwen.

Das 1942 eingeführte System hat bis Kriegsende trotz der Absicht seiner Erweiterung auf das ganze Reichsgebiet ohne wesentliche Änderungen weiter gegolten. Die Erweiterung wurde wegen negativer Beurteilungen dieser Regelung im Hinblick auf die Leistungsfähigkeit der polnischen Beschäftigten, wie auch im Hinblick auf die Möglichkeiten der Rekrutierung der Polen aus dem Generalgouvernement in die Arbeit in den eingegliederten Ostgebieten verhindert.

Die durch den Erlass des Reichsarbeitsministers vom 26. August 1942 eingeführte Regelung bedeutete den kompletten Ausschluss der Polen aus der Sozialversicherung. Sie wurden auch aus der deutschen Sozialversicherung ausgeschlossen. Zwar galt für sie in gewissem Umfang die Verordnung über die Einführung der Reichsversicherung in den eingegliederten Ostgebieten vom 22. Dezember 1941, jedoch nur entsprechend und nur insoweit als der Erlass nicht anderes bestimmte. Praktisch waren es Regelungen aus dem Bereich der Organisation. In den damaligen Kommentaren wurde das für Polen eingeführte System mit der öffentlichen Fürsorge verglichen. In diesem Zusammenhang ist darauf hinzuweisen, dass sowohl im deutschen als auch in anderen damaligen Systemen im Rahmen der öffentlichen Fürsorge keine Beiträge erhoben wurden. Darüber hinaus räumte z.B. das polnische Fürsorgegesetz vom 16. August 1923 den Gemeindeeinwohnern das Recht auf eine dauerhafte Unterstützung von Seiten der Gemeinde ein.

76 Oberschlesisches Institut für Wirtschaftsforschung (Hrsg.), Die Bedeutung des Polen-Problems (Fußn. 75), S. 136. 
In der Sozialversicherung wie auch in der Fürsorge wurde der Familienstand berücksichtigt. Aus dem für Polen eingeführten System wurden Leistungen dieser Art weitgehend entfernt. Die Grundsätze der Rassenpolitik haben in diesem Bereich einen besonders deutlichen Ausdruck gefunden. Insgesamt kann gesagt werden, dass Polen nicht nur aus der Sozialversicherung in der damaligen Prägung, sondern auch aus der Fürsorge verdrängt wurden.

\section{Die arbeits- und sozialversicherungsrechtliche Lage der polnischen Ar- beitnehmer in Deutschland 1946-1994}

\section{Die Nachkriegszeit}

Eine der Konsequenzen des 2. Weltkrieges war die Teilung Deutschlands in zwei Staaten: die Bundesrepublik Deutschland (21. September 1949) und die Deutsche Demokratische Republik (7. Oktober 1949), die damals zu zwei feindlichen politischen Blocks gehörten. Die diplomatischen Beziehungen zwischen Polen und der BRD wurden erst 1972 aufgenommen, nach Unterzeichnung des Abkommens über Grundlagen der Normalisierung der gegenseitigen Beziehungen (1970).

Nach dem Krieg erhielt der größte Teil der sich in Deutschland befindlichen ehem. polnischen Zwangsarbeiter und Kriegsgefangenen in den westlichen Besatzungszonen anfangs den Status von displaced persons. Unter diesem Begriff verstanden die Alliierten „Zivilpersonen, die sich aus Kriegsfolgegründen außerhalb ihres Staates befinden; die zwar zurückkehren oder eine neue Heimat finden wollen, dieses aber ohne Hilfestellung nicht zu leisten vermögen". 77 Von den Ende September 1945 in Westdeutschland verbliebenen displaced persons waren 66\% Polen. In der Folgezeit kamen diese entweder nach Polen zurück, wanderten in den Westen aus oder in Deutschland.

Das Sonderrecht für Ausländer hatten die Alliierten mit dem Kontrollratsgesetz Nr. 1 vom 20 September 1945 außer Kraft gesetzt. Weiter galten jedoch die Verordnung über ausländische Arbeitnehmer vom 23. Januar 1933 wie auch die Ausländerpolizeiverordnung vom 22. August 1938. Die ersten Rekrutierungen zur Arbeit in der BRD begannen in den 50er Jahren. Sie vollzogen sich auf der Rechtsgrundlage von Arbeitsvereinbarungen, welche die BRD mit Italien 1955, Spanien und Griechenland 1960 und der Türkei 1961 abgeschlossen hatte. ${ }^{78}$

Was die Beschäftigung der Polen in der BRD anbetrifft, so war der polnische Staat anfangs nicht daran interessiert, sowohl im Hinblick auf die nahe Vergangenheit, die

77 Herbert/Hunn/Hoffmann, Beschäftigung, soziale Sicherung und soziale Integration von Ausländern, Geschichte der Sozialpolitik in Deutschland seit 1945, B. 2/1, S. 818 ff.

78 Eichenhofer, Internationale Sozialpolitik, in: Geschichte der Sozialpolitik in Deutschland seit 1945, Bd. 5 1966-1974, 2006, S. 933. 
angespannte politische Lage zwischen beiden Staaten, aber auch deshalb, weil im Lande alle verfügbaren Arbeitskräfte für die Durchführung der Aufgaben des Sechs-JahreWirtschaftsplans (1950-1956) benötigt wurden. Außerdem war das Ausland für Polen, mit Ausnahme der sozialistischen Länder, aus politischen Gründen überhaupt nicht zugänglich. Dennoch, u.a. wegen des Devisenmangels und um die wirtschaftlichen Kontakte mit dem Westen zu erhalten, bediente sich Polen der im Bereich der internationalen Zusammenarbeit bekannten Institution der Werkverträge zur Durchführung von Bauarbeiten im Ausland. Diese Arbeiten führten polnische Firmen in der BRD mit Hilfe von polnischen Arbeitnehmern, unter Anwendung des polnischen Arbeitsrechts, durch. Die Einzelheiten der mit polnischen Firmen in der damaligen Zeit abgeschlossenen Arbeitsverträge sind nicht bekannt. Diese Form von Beschäftigung polnischer Arbeiter im Ausland hat sich etabliert und später ihre rechtliche Form in einer Verordnung des Ministerrats aus dem Jahre 1974 gefunden.

Was die gegenseitigen Beziehungen zwischen Polen und der BRD im Bereich der sozialen Sicherheit anbelangt, so wurden in dieser Hinsicht in der Zeit bis 1970 keine offiziellen Kontakte aufgenommen. Die schwierige Aufgabe, die durch die Zwangsarbeit, durch territoriale Verschiebungen nach dem Krieg, aber auch noch durch Sachverhalte aus früheren Zeiten entstandenen Probleme der Gewährung einer sozialen Sicherung zu lösen, hat Polen auf sich genommen. Gemäß $§ 1$ der Verordnung des Ministers für Arbeit und Sozialfürsorge vom 3. April $1950^{79}$ stehen Personen mit polnischer Staatsangehörigkeit, die in Polen wohnen und Rechtsansprüche gegen ausländische Sozialversicherungsträger erlangt haben, unter in der Verordnung vorgesehenen Bedingungen Leistungen der polnischen Sozialversicherung zu. In $\S 2$ der Verordnung wurde ihr Anwendungsbereich auf Ansprüche begrenzt, die während einer Beschäftigung außerhalb der gegenwärtigen Grenzen Polens entstanden waren. Im Ausland zurückgelegte Versicherungszeiten wurden den polnischen gleichgestellt. Als Versicherungszeiten im Sinne der Verordnung wurden ausdrücklich die Zeiten einer Beschäftigung im Reich und in den zwischen dem 1. September 1939 und dem 31. Dezember 1946 eingegliederten Ostgebieten wie auch einer Beschäftigung im Deutschen Reich genannt.

Ein Zeichen für die Aufnahme zwischenstaatlicher Beziehungen im Bereich der sozialen Sicherheit noch vor der Aufnahme diplomatischer Beziehungen zwischen Polen und der BRD war die Verwaltungsvereinbarung vom 20. Juli 1967, abgeschlossen zwischen der polnischen Sozialversicherungsanstalt und den Versorgungsämtern in Ravensburg, Münster, Gelsenkirchen und Hamburg über die Gewährung von Leistungen an invalide ehemalige Wehrmachtsangehörige, ihre Familien und gleichgestellte

79 Rozporządzenie Ministra Pracy i Opieki Społecznej w sprawie uznania uprawnień, nabytych w zagranicznych instytucjach ubezpieczenia społecznego (Verordnung des Ministers für Arbeit und Sozialfürsorge über in ausländischen Sozialversicherungsanstalten erworbene Ansprüche), Dz. U. R. P, Nr. 17, Pos. 148. 
Personen. ${ }^{80}$ Gegenstand dieser Vereinbarung war insbesondere die Überweisung von Geldleistungen nach Polen. Außerdem wurden Angelegenheiten einer ärztlichen Begutachtung der Berechtigten und der damit verbundenen Kosten, aber auch die Vermittlung durch die polnische Sozialversicherungsanstalt bei Antragstellung vereinbart. Andere Verwaltungsvereinbarungen hatten die Zollfreiheit für die von der Bundesrepublik Deutschland im Rahmen der Kriegsopferversorgung nach Polen geleisteten orthopädischen Hilfsmittel zum Gegenstand. Dieses Prinzip wurde auf weitere für Kriegsbehinderte zu liefernde Gebrauchsgegenstände erstreckt. ${ }^{81}$

Die Deutsche Demokratische Republik und die Volksrepublik Polen schlossen am 13. Juli 1957 in Warschau ein Abkommen über die Zusammenarbeit im Bereich der Sozialpolitik ab. In dem für uns interessanten Bereich wurde im Abkommen zunächst der Grundsatz der Gleichbehandlung in der arbeitsrechtlichen Behandlung und in der Sozialversicherung formuliert (Art. 2). Weitere Bestimmungen hatten die Sozialversicherung zum Gegenstand. Die gegenseitigen Beziehungen in diesem Bereich basierten auf dem Eingliederungsprinzip.

\section{Die Zeit der Normalisierung 1970-1989}

Der Beginn einer zweiten Phase in den Beziehungen zwischen der Bundesrepublik Deutschland und Polen kann mit der Unterzeichnung des Warschauer Vertrages über die Grundlagen der Normalisierung der gegenseitigen Beziehungen im Jahre 1970 und den alsbald aufgenommenen diplomatischen Beziehungen zwischen den beiden Staaten datiert werden. In dieser Zeit etablierte sich im Bereich der Beschäftigung polnischer Arbeitnehmer in Deutschland die schon bekannte Form der Beschäftigung mittels der mit polnischen Firmen abgeschlossenen Werkverträge. Die Rechte und Pflichten der durch die polnischen Firmen beschäftigten Arbeiter wurden in einer Verordnung des Ministerrats vom 27. Dezember 1974 über einige Rechte und Pflichten der ins Ausland zur Durchführung von Exportbauvorhaben gesandten Arbeitnehmer, ergänzt durch den Beschluss Nr. 71 des Ministerrats über die Prinzipien der Entlohnung und die Zuerkennung anderer mit der Arbeit verbundener Leistungen, geregelt. Für eine Beschäftigung in der BRD schloss das polnische Unternehmen mit dem Arbeitnehmer einen zusätzlichen befristeten Arbeitsvertrag. Die Entlohnung wurde im Arbeitsvertrag bestimmt, weitere wichtige Angelegenheiten der Beschäftigung (Erholungsurlaub, Arbeitszeit, Familienfahrten, Devisenanteil an der Entlohnung) waren in den bereits zitierten Rege-

80 S. den Text dieser Vereinbarung bei Sobczak, Ubezpieczenie społeczne w umowach międzynarodowych - zbiór tekstów (Sozialversicherung in internationalen Vereinbarungen - eine Textsammlung), 1995, S. 322.

81 Eichenhofer, Internationale Sozialpolitik (Fußn. 78), S. 938. 
lungen enthalten. ${ }^{82}$ Ansonsten war eine legale Beschäftigung polnischer Arbeiter in der Bundesrepublik weiterhin ausgeschlossen.

Die aus der zunehmenden Zahl der in der Bundesrepublik im Rahmen der Werkverträge beschäftigten polnischen Arbeitnehmer resultierenden sozialversicherungsrechtlichen Probleme sollten durch ein Abkommen geregelt werden. So entstanden auch die ersten förmlichen sozialversicherungsrechtlichen Beziehungen zwischen der Bundesrepublik und der Volksrepublik Polen, die mit dem Vertrag vom 25. April 1973 über die Sozialversicherung der Arbeitnehmer, die vorübergehend in das andere Land entsandt worden waren (sog. Entsendeabkommen), begonnen haben. ${ }^{83}$ Das Entsendeabkommen betraf die Kranken-, Unfall- und Rentenversicherung der durch den Arbeitgeber in das Gebiet des anderen Vertragsstaates vorübergehend entsandten Arbeitnehmer und regelte die entsprechende Durchführung. Die Vertragspartner vereinbarten, dass die entsandten Arbeitnehmer 24 Monate den Vorschriften des Entsendestaates unterlagen. Im Vertrag wurde die schon seit langem in der Sozialversicherung bekannte Figur der Eingliederung angewandt. Die VR Polen strebte nämlich an, dass für ihre in der Bundesrepublik Deutschland tätigen Arbeitnehmer keine Beiträge zur deutschen Sozialversicherung erhoben werden sollten. ${ }^{84}$ Die soziale Sicherheit der entsandten polnischen Arbeitnehmer richtete sich grundsätzlich nach dem polnischen Recht. Dennoch wurde den entsandten Arbeitnehmern das Recht eingeräumt, sich freiwillig bei der zuständigen AOK zu versichern, gegen einen herabgesetzten Beitrag. Bei Arbeitsunfällen wurde den Arbeitnehmern gemäß den für Inländer geltenden Bestimmungen erste Hilfe geleistet.

Der zweite wichtige Rechtsakt im Bereich der seit dem 2. Weltkrieg weitgehend ungeregelten sozialversicherungsrechtlichen Beziehungen zwischen der VR Polen und der BRD war das deutsch-polnische Abkommen über die Renten- und Unfallversicherung vom 9. Oktober 1975. ${ }^{85}$ Es wurde im Rahmen eines Gesamtpakets aus drei weiteren Vereinbarungen abgeschlossen: im Einzelnen die Vereinbarung über die wechselseitige Abgeltung aller Ansprüche auf dem Gebiet der Renten- und Unfallversicherung, über die Gewährung eines Finanzkredits und über die Ausreise von 120.000 bis 125.000 Deutschen im Laufe der vier nächsten Jahre.

Ich beschränke mich an dieser Stelle auf das Rentenabkommen, das auf dem Prinzip der Eingliederung basierte. ${ }^{86}$ Danach hatte jeder Berechtigte seine Rente vom Versicherungsträger des Staates, in dem der Berechtigte wohnte, nach den für diesen Träger geltenden Vorschriften zu erhalten (Art. 4 des Abkommens). Bei der Feststellung der Ren-

82 Florek, Die Beschäftigung der polnischen Arbeitnehmer im Ausland und der Ausländer in Polen, Europäische Integration und nationale Rechtskulturen, Referate des 13. Symposiums der Alexander von Humboldt-Stiftung in Bamberg, 1995, S. 435 ff.

83 Dz. U. 1974, Nr. 42, Pos. 250.

84 Eichenhofer, Internationale Sozialpolitik (Fußn. 78), S. 939.

85 Dz. U. 1976, Nr. 16, Pos. 102.

86 Haas, Die Grundprinzipien, BArBl. 1976, S. 211 ff.; Eichenhofer, Internationale Sozialpolitik (Fußn. 78), S. 878 ff. 
te berücksichtigte dieser Träger die Versicherungszeiten im anderen Vertragsstaat so, als ob sie im eigenen Land zurückgelegt worden wären.

Renten standen nur für die Zeit zu, in der die betreffende Person in dem Gebiet des Staates wohnte, dessen Versicherungsträger die Rente festgestellt hatte. Beim Verlegen des gewöhlichen Aufenthaltes durch den Rentner in das Gebiet des anderen Staates sollte die Zahlung der Rente eingestellt werden.

Das Abkommen begründete Rechte und Leistungsverpflichtungen nur für einen bestimmten Zeitraum nach seinem Inkrafttreten. Allerdings sollten bereits bewilligte Renten weiter in das andere Land gezahlt werden (Art.15, Art.16).

Das mit diesem Abkommen gewählte Eingliederungsprinzip war in Deutschland im Hinblick darauf, dass mit anderen Staaten vornehmlich Abkommen nach dem Leistungsexportprinzip geschlossen worden waren, Gegenstand von vielen Diskussionen. Es wurden folgende Gründe für das Eingliederungsprinzip genannt:

- Die wechselvolle Vergangenheit in den deutsch-polnischen Verhältnissen, die eine Zuordnung der Sozialversicherungstatbestände nahezu unmöglich machten.

- Das Abkommen behielt im Grunde die zu jener Zeit bestehende Situation bei, denn das Eingliederungsprinzip wurde nach innerstaatlichem Recht gegenüber den Deutschen, die aus Polen kamen, und gegenüber den Deutschen, die in Polen verblieben, bereits weitgehend angewandt.

- Es war sozialpolitisch sinnvoll, weil es überhöhte Renten auf der einen und unzulängliche Renten auf der anderen Seite vermied, wenn das Lebensniveau in den beiden vertragschließenden Staaten sehr unterschiedlich war.

Im polnischen Schrifttum wurde die Anwendung des Art. 16 des Abkommens kritisiert. ${ }^{87}$ Gemäß dieser Bestimmung berührte dieses Abkommen die Zahlung der Renten auch für die Zeit nach seinem Inkrafttreten nicht, soweit Renten der Renten- oder Unfallversicherung der BRD für den Kalendermonat, der dem Inkrafttreten des Abkommens voranging, an Personen im Gebiet der VR Polen zu zahlen waren.

Es handelte sich um die Frage, wie die Gebiete östlich der Oder-Neiße im Hinblick auf die Vorschriften des damaligen Auslandsrentenrechts ( $§ 1318$ ff. RVO) über das Ruhen von Renten zu betrachten waren. Die in der Rechtsprechung des BSG und des BVG ausgearbeiteten Rechtsfiguren des Inlands, des Auslands und der „Gebiete außerhalb des Geltungsbereiches dieses Gesetzes", wonach die an Polen in Potsdam zuerkannten Gebiete nicht als Ausland, sondern als „Gebiete außerhalb des Geltungsbereiches dieses Gesetzes" zu betrachten waren, ${ }^{88}$ wurde von der polnischen Seite als Infragestellung des Warschauer Abkommens aus dem Jahre 1970 betrachtet und deshalb kritisiert. Die praktischen Folgen waren, dass Personen, die in Polen in den Gebieten an der Oder und der Neiße wohnten, keine Rentenleistungen gezahlt bekamen, weil sie

87 S. Bundesverfassungsgericht, Entscheidung vom 26. Februar 1980 (1 BvR 195/77); Barcz, Stosunki rentowe PRL-RFN. Zagadnienia artykulu 16 umowy z 1975 roku (Die Rentenverhältnisse VRPBRD. Probleme des Art. 16 des Vertrags aus dem Jahre 1975), 1986, S. 9 ff.

88 Barcz, Stosunki rentowe PRL-RFN (Fußn. 87), S. 9 ff. 
nicht im Ausland wohnten, dagegen Berechtigte mit gewöhnlichem Aufenthalt in Zentralpolen ihre Rente in voller Höhe erhielten (soweit sie auf den im Bundesgebiet zurückgelegten Beitragszeiten beruhte).

\section{III. Übergang zur Zusammenarbeit}

Eine weitere Etappe in den Beziehungen zwischen der Bundesrepublik Deutschland und der Republik Polen im Bereich der Beschäftigung der polnischen Arbeitnehmer war die Zeit nach 1989, also nach dem Übergang Polens von einer sozialistischen Gesellschaftsordnung zu einer demokratischen Marktwirtschaftsordnung.

Die Intensivierung der Zusammenarbeit im Bereich der Beschäftigung lag im beiderseitigen Interesse. Die BRD konnte die regionalen und sektoralen Bedürfnisse an Arbeitskräften so besser befriedigen; für Polen bedeutete es, wegen der steigenden Arbeitslosigkeit im Lande als Folge der eingeführten Wirtschaftsreformen, eine Beschäftigung zu erhalten und den Lebensstandard verbessern zu können.

Es bestand ein Trend zur Aufnahme einer Beschäftigung in Deutschland der darin resultierte, dass außer den zugelassenen Formen einer legalen Beschäftigung auch illegale Beschäftigung (sog. Schwarzarbeit) stattfand. Wie es in einer freiheitlichen Marktwirtschaft vorkommt, lag das Interesse an Schwarzarbeit auch bei den deutschen Arbeitgebern selbst. Die genauen Zahlen der illegalen Beschäftigung sind nicht bekannt; diese liegen auch nicht im Zentrum unserer Erwägungen. Was die legalen Formen einer Beschäftigung betrifft, so konnte sie in drei Formen verwirklicht werden:

- im Rahmen eines Werkvertrags zwischen einem polnischen Arbeitgeber und einem deutschen Unternehmer für eine vorübergehende Tätigkeit im Bausektor,

- im Rahmen der sog. Gastarbeiterverträge und

- durch Beschäftigung im Rahmen von Saisonarbeiten.

Die Anwendung der schon in den 1960er Jahren praktizierten Form von Werkverträgen wurde, im Hinblick auf die Lage auf dem Arbeitsmarkt und die Bestrebungen um eine Einschränkung der illegalen Beschäftigung, sehr formalisiert. Rechtsgrundlage für die Tätigkeit polnischer Unternehmer in Deutschland war die Vereinbarung zwischen der Regierung der Bundesrepublik Deutschland und der Regierung der Republik Polen über die Entsendung von Arbeitnehmern polnischer Unternehmen zur Ausführung von Werkverträgen vom 31. Januar 1990.89

Als rechtliche Voraussetzungen für die Tätigkeit von polnischen Arbeitnehmern in polnischen Unternehmen wurden vorgesehen:

- das Vorhandensein eines Werkvertrages, den das polnische Unternehmen in Deutschland ausführt,

89 RGBl. II, 1990, S. 602, mit späteren Änderungen. 
- Nichtüberschreitung des jährlich festgelegten Arbeitnehmerkontingents für diese Form der Beschäftigung (in den Jahren 1990-1993 waren es durchschnittlich 35.000 Arbeitnehmer),

- Beschäftigung von Facharbeitern,

- die sich aus den Werkverträgen ergebende Entlohnung (einschließlich des Teils, der wegen der auswärtigen Beschäftigung gezahlt wurde) entsprach dem Lohn, welchen die deutschen Tarifverträge für vergleichbare Tätigkeiten vorsahen,

- Begrenzung der Arbeitserlaubnis auf zwei Jahre und eine Karenzzeit vor der nächsten Beschäftigung.

Um einer Arbeitnehmerüberlassung an den deutschen Auftraggeber vorzubeugen, wurde das gesamte behördliche Verfahren in Polen und in Deutschland nur von den polnischen Unternehmern betrieben (u.a. der Abschluss des Werkvertrages, der Antrag auf Zuteilung von Plätzen aus dem Kontingent durch eine polnische Auswahlkommission und der Antrag auf Zuteilung der Arbeitserlaubnis durch das Landesarbeitsamt). ${ }^{90}$

Die Beschäftigung als Gastarbeiter erfolgte gemäß einer Vereinbarung vom 7. Juni 1990 zwischen den Regierungen der Bundesrepublik und der Republik Polen. Jährlich konnten 1.000 Personen als Gastarbeiter beschäftigt werden. Den Gastarbeiterstatus konnten polnische Arbeitnehmer im Alter zwischen 18 und 35 erhalten, die eine berufliche Ausbildung hatten und für die die Beschäftigung einer Verbesserung ihrer fachlichen und sprachlichen Qualifikationen dienen sollte. Die Beschäftigungsdauer wurde auf ein Jahr begrenzt; jedoch mit der Möglichkeit einer Verlängerung auf bis zu 18 Monate. Entlohnung und andere Arbeitsbedingungen sollten denen entsprechen, die in den Tarifverträgen wie auch in arbeits- und sozialrechtlichen Vorschriften vorgesehen waren.

Die Beschäftigung im Rahmen von Saisonarbeiten wurde formell aufgrund einer Vereinbarung der deutschen und polnischen Arbeitszentralverwaltungen zugelassen. Sie umfasste die Beschäftigung in Betrieben der Land- und Forstwirtschaft, in der Gastronomie und im Hotelgewerbe, sowie in der Gemüse- und Obstverarbeitung. Die Beschäftigung in dieser Form wurde nicht kontingentiert. In den Jahren 1991-1995 nahmen insgesamt 666.572 polnische Arbeitnehmer eine solche Beschäftigung auf. Die Lohnund Arbeitsbedingungen waren mit denen der deutschen Arbeitnehmer vergleichbar. ${ }^{91}$

90 Näher dazu Kaligin, Tätigkeit von Bauunternehmen aus Polen und der CSFR in Deutschland, NZA 1992, S. $1111 \mathrm{ff}$.

91 Tegtmayer, Bilans współpracy polsko-niemieckiej w dziedzinie polityki społecznej i migracji (Eine Bilanz der polnisch-deutschen Zusammenarbeit im Bereich der Sozialpolitik und Migration), in: von Maydell (Hrsg.), Lad społeczny w Polsce i Niemczech, Księga pamiątkowa poświęcona Czesławowi Jackowiakowi (Die Sozialordnung in Polen und Deutschland in einem zusammenwachsenden Europa, Gedächtnisschrift für Czeslaw Jackowiak), 1999; Marek, Wyjazdy zarobkowe Polaków do Republiki Federalnej Niemiec (Erwerbsreisen der Polen in die Bundesrepublik Deutschland), 2001, H. 8, S. $29 \mathrm{ff}$. 
Einige Polen in den westlichen Grenzgebieten nahmen eine Tätigkeit als Grenzgänger in Werkstätten oder Gärtnereien auf. Schätzungsweise waren es im Jahre 20014.000 bis 4.500 Personen.

Das durch Polen mit den Europäischen Gemeinschaften 1994 abgeschlossene Assoziierungsabkommen brachte im Bereich der Formen der Beschäftigung polnischer Arbeitnehmer in Deutschland keine Änderungen. Die polnischen Bemühungen in das Assoziierungsabkommen den Grundsatz der Freizügigkeit polnischer Bürger innerhalb der Gemeinschaften im gleichen Umfang einzuführen, wie er im Art. 48 des Vertrags über die Europäische Wirtschaftsgemeinschaft formuliert war, sind gescheitert. 92 Die Mitgliedstaaten haben sich in Art. 41 des Abkommens lediglich verpflichtet, die bestehende Erleichterungen beim Zugang zu den Arbeitsmärkten weiter zu erhalten bzw. falls noch keine entsprechenden Verträge abgeschlossen worden waren, den Abschluss solcher wohlwollend zu überlegen.

Im Bereich der Sozialversicherung wurde am 19. Oktober 1990 ein neues Abkommen über die soziale Sicherheit paraphiert und am 8. Dezember 1990 in Warschau unterschrieben. 93

In sachlicher Hinsicht bezieht sich das Abkommen auf deutscher Seite auf die Kranken-, Unfall- und Rentenversicherung und auf polnischer Seite auf die Leistungen der Arbeitnehmer bei Krankheit und Mutterschaft, die Unfallversicherung und die Altersversorgung der Arbeitnehmer. Eine detaillierte Besprechung dieses Abkommens, das sehr umfangreich ist, würde den Rahmen dieses Aufsatzes sprengen und ist, im Hinblick auf das Thema dieses Aufsatzes, auch nicht notwendig.

Das Abkommen baute grundsätzlich auf den in den damaligen europarechtlichen Vorschriften enthaltenen Regeln der Koordination von Systemen der sozialen Sicherheit auf. Insbesondere beruht es auf dem Grundsatz der Personengleichstellung und regelt, dass die Staatsangehörigen der Vertragsparteien bei Anwendung der Rechtsvorschriften einer Vertragspartei einander gleichstehen, wenn sie sich gewöhnlich im Gebiet einer Vertragspartei aufhalten. ${ }^{94}$ Das wichtigste Merkmal des Abkommens ist seine Ausgestaltung nach dem Leistungsexportprinzip. Im Bereich der Rentenversicherung bedeutet es, dass die nationalen Voraussetzungen für den Anspruch auf eine Rente auch unter Zusammenrechnung der nach den deutschen oder polnischen Rechtsvorschriften zurückgelegten oder anrechnungsfähigen Versicherungszeiten geprüft werden. ${ }^{95}$ Art. 17 Abs. 1 des Abkommens besagt, dass, wenn in beiden Vertragsstaaten Versicherungszeiten vorhanden sind, für den Erwerb des Leistungsanspruchs nach den Rechtsvorschrif-

92 Szurgacz, Sprawy pracy i sprawy socjalne w Układzie Europejskim ustanawiającym Stowarzyszenie Polski z Wspólnotami Europejskimi (Arbeits- und sozialrechtliche Angelegenheiten im Abkommen über die Assoziierung Polens mit den Europäischen Gemeinschaften), in: ders. (Hrsg.), Europejskie prawo pracy i prawo socjalne a prawo polskie (Das europäische Arbeits- und Sozialrecht und das polnische Recht), 1998, S. 113.

93 Dz. U. 1991, Nr. 108, Pos. 468; BGB1. II, 1991, S. 741.

94 Polster, Das neue Abkommen mit Polen, Deutsche Rentenversicherung 1991, S. 264.

95 Polster, Das neue Abkommen (Fußn. 94). 
ten eines Vertragsstaats auch die Versicherungszeiten berücksichtigt werden, die nach den Rechtsvorschriften des anderen Vertragsstaats anrechnungsfähig sind und nicht in dieselbe Zeit fallen. Was die Rentenberechnung betrifft, wurde die Methode Pro-ratatemporis angewandt. Sind gemäß Art. 18 Abs. 1 die Voraussetzungen für den Erwerb des Leistungsanspruchs erfüllt, so erbringt der zuständige Träger eine Rente, deren Höhe grundsätzlich dem Verhältnis entspricht, in dem die nach seinen Rechtsvorschriften zurückgelegten Versicherungszeiten zu den zusammengerechneten Versicherungszeiten stehen. Hierzu ermittelt er den von ihm zu zahlenden Rentenbetrag nach den für ihn geltenden Rechtsvorschriften.

Gemäß Art. 6 erhalten Sachleistungen der Krankenversicherung auch Arbeitnehmer die in einem Vertragsstaat versichert sind und sich im anderen Vertragsstaat als entsandte Arbeitnehmer aufhalten, Familienangehörige von Arbeitnehmern (ausgenommen von entsandten Arbeitnehmern) und Grenzgänger. Dies gilt jedoch nur für Personen, bei denen der Versicherungsfall während des vorübergehenden Aufenthalts im anderen Vertragsstaat eintritt, und nur, wenn sie wegen ihres Zustands sofort Leistungen benötigen. Weitere Bestimmungen präzisieren diesen Grundsatz und weisen auf die für die Erbringung der Leistungen zuständigen Träger hin. Geldleistungen der Krankenversicherung sind nach den Vorschriften des einen Staates zu zahlen, wenn sie sich im Gebiet des anderen Staates aufhalten und Anspruch auf Sachleistungen haben.

Im Bereich der Sozialversicherung hat die Assoziierung Polens mit der EG für die Beziehungen zwischen Polen und Deutschland keine Veränderungen gebracht. Gemäß Art. 38 des Assoziierungsabkommens werden im Hinblick auf die Koordinierung von Systemen sozialer Sicherheit gegenüber polnischen Bürgern, die sich auf den Territorien der Mitgliedsstaaten legal aufhalten, die Grundsätze der Zusammenrechnung der in den Mitgliedstaaten abgelegten Beitrags-, Beschäftigungs- und Aufenthaltszeiten und des Leistungsexports eingeführt.

\section{Schlussbemerkungen}

Die rechtliche Lage der polnischen Arbeitnehmer während einer über hundert Jahre langen Geschichte ihrer Beschäftigung in Deutschland konnte hier nur skizzenhaft dargestellt werden. Aus der vorliegenden Darstellung ist ersichtlich, dass die arbeits- und sozialrechtliche Lage der polnischen Arbeitnehmer Wandlungen unterlag. In einigen Zeitabschnitten haben nationalpolitische Faktoren großen Einfluss auf ihre rechtliche und faktische Lage ausgeübt. Verbunden mit Interessen der Rüstungs- und Kriegswirtschaft führten sie während des 1. Weltkrieges dazu, dass eine freiwillige Beschäftigung in eine Zwangsbeschäftigung umgestaltet wurde. In den Jahren 1939-1945 führte der

Nationalsozialismus für polnische Arbeiter eine besondere Rechtsordnung ein: das Recht der Zwangsarbeit, das der Realisierung des Zieles der Umwandlung der Polen in 
Sklaven des Großdeutschen Reiches dienen und Nachwirkungen über die Zeit des Krieges hinaus haben sollte.

Dagegen konnten sich in anderen Zeitabschnitten, in denen nationalpolitische Faktoren in geringerem Ausmaß oder gar nicht auftraten, im Arbeitsrecht und in der Sozialversicherung Grundsätze einer Gleichbehandlung mit den deutschen Arbeitnehmern und der Nichtdiskriminierung stufenweise durchsetzen. Dies betrifft die Zeit der Weimarer Republik, und nach dem 2. Weltkrieg die Zeit der Bundesrepublik Deutschland sowie die Beschäftigung in der Deutschen Demokratischen Republik. In diesen Zeitabschnitten ist die Zusammenarbeit der deutschen und polnischen Sozialversicherungsträger hervorzuheben. Sie führte zum Abschluss zwischenstaatlicher Verträge, welche die Ungleichheiten und die Auswirkungen der Territorialität in der sozialen Sicherheit mildern sollten. 


\title{
Unionsrechtliche Vorgaben für die Arbeitnehmerfreizügigkeit
}

\author{
Ulrich Becker
}

A. Einleitung $\quad 65$

B. Eigenheit der Arbeitnehmerfreizügigkeit $\quad 67$

C. Voraussetzungen der Arbeitnehmerfreizügigkeit 68

D. Inhalt der Rechte auf und im Aufenthalt 70

E. Grenzen der Arbeitnehmerfreizügigkeit 72

F. Schlußwort $\quad 74$

\section{A. Einleitung}

Nach dem Fall der Berliner Mauer und der Auflösung der politischen Nachkriegsblöcke in Europa standen nicht nur die mittel- und osteuropäischen Staaten vor einer Neuordnung, sondern auch die Europäische Union. Schnell war klar, daß es einen zweiten Europäischen Wirtschaftsraum nicht geben sollte, sondern die Grenzen der europäischen Integration neu zu ziehen waren. Jedoch weckte die politisch gewollte und erforderliche Erweiterung der Europäischen Union angesichts deren Größe und der zumindest zu Beginn bestehenden wirtschaftlichen Unterschiede nicht nur Hoffnungen, sondern auch Befürchtungen. Expertisen wurden geschrieben: über die Folgen einer Arbeitskräftewanderung für die in den alten Mitgliedstaaten bestehenden sozialen Sicherungssysteme ${ }^{1}$ ebenso wie über die europarechtliche Zulässigkeit der Beschränkung der Freizügigkeit ${ }^{2}$. Man entschied sich für Übergangsregelungen ${ }^{3}$, die bei früheren Beitrit-

1 Vgl. Sinn, Die Osterweiterung der EU und die Zukunft des Sozialstaates, in: Leibfried/Wagschal (Hrsg.), Der deutsche Sozialstaat, Bilanzen - Reformen - Perspektiven, 2000, S. 474, 477 ff.; ders., Introduction, in: Social Union and Migration, CESifo No. 3/2004, S. 4, 5.

2 Becker, EU-Erweiterung und differenzierte Integration, 1999.

3 Art. 24 i.V.m. Anhang XII der Akte über die Bedingungen des Beitritts der Tschechischen Republik, der Republik Estland, der Republik Zypern, der Republik Lettland, der Republik Litauen, der Republik Ungarn, der Republik Malta, der Republik Polen, der Republik Slowenien und der Slo- 